XII.

\title{
Über sensorische Nerven und periphere Sensibilitäten.
}

\author{
Von \\ Page May, London. \\ (Mit 6 Abbildungen anf Tafel I-IV.)
}

Literatur.

1. Alrutz, Smärtsinnet. Upsala 1901.

2. Derselbe, Studien auf dem Gebiete der Temperatursinne. Skand. Archiv f. Physiol. 1897. 7. 333 .

3. Derselbe, Studien auf dem Gebiete der Temperatursinne. Skand. Archiv f. Physiol. 1897. 7. 326.

4. Alsberg, Untersuchungen über den Raum- und Temperatursinn. Dissertat. Marburg 1863.

5. Bader, Wuudts philosophische Studjen. 1903. 18. 452.

6. Bayliss, Further researches on antidromie nerve-impulses. Journ. physiol. 1902. 28. Nr. 4.

7. Derselbe, Further researches on antidromie nervo-impulses. Journ. physiol. 1901. 26. 175, Nr. $3-4$.

8. v. Bechterew, Ein neues Algesimeter. Neurol. Zentralbl. 1899. 18. 286.

9. B lix, Upsala Läkareförenings förh. 1882--83. 18.

10. Derselbe, Experimentelle Beiträge zur Lösung der Frage über die spezifische Energie der Hautnerven. Zeitschr. f. Biol. 1884. 20. 141.

11. Derselbe, Experimentelle Beiträge zur Lösung der Frage üher die sperifische Energie der Hautnerven. Zeitsehr. f. Biol. 1885. 21. 158.

12. Derselbe, Experimentelle Beiträge zur Lösung der Frage über die spezifische Energie der Hautnerven. Zeitschr. f. Biol. 1885. 21. 145.

13. Brissaud, Leçons sur les maladies nerveuses; deuxième série. 1899.

14. Brown-Séquard, Journ. de. Physiol. 1864. 6 124, 232, 581.

15. Buzzard, Uniradic. Palsies of the Brachial Plexus. Brain. 1902. Pt. 108. 299.

16. Camus et Sézary, Les Radiculites. La Presse méd. 24 Août. 1907.

17. Charcot, Clinique des Maladies nervenses. T. 1. 333.

18. Darwin, Erasmus, Zoonomia or the laws of Organic Life. London 1794.

19. Déjérine, Revue Neurolog. 15 Juill. 1908.

20. Dersel be, Semeiologie du Syștème nerveux. Traité de Pathologie générale de Bouchard. p. 773 et 937. 
21. Déjérine et Egger, Un cas de névrite radiculaire. Revue neurologique. 15 Juin. 1904.

22. Delille et Camus, Un eas de zona rigoureusement radiculaire. Revue nenrologique. 1902, Tome 10.

23. Dessoir, Über den Hautsinn. Arch. f. Anat. u. Physiol. (Physiol. Abt.) 1892, 250.

24. Donalds on, Mind. 1885. 10.

25. Fechuer, Elemente der Psychophysik. 1860. 1. 201.

26. Féré, Note sur quatre cas de Zona. Revue de Méd. 1890. 5. 393.

27. v. Frey, Berichte der Math. Phys. Klasse d. königl. sächs. Gesellsch. d. Wissensch. zn Leipzig 1894. 1895.

28. Derselbe, Leipziger Ber. 1895. 172.

29. Derselbe, Leipziger Abh. 1896.

30. Derselbe, Untersuchungen über die Sinnesfunktion der menschlichen Haut. Ahh. d. math. phys. Klasse d. königl. sächs. Gesellsch. d. Wissensch. 1896. 23. 175.

31. Goteh and Horsley, On the mammal nerv. syst., its functions and their localisation determined by an elect. method. Phil. Trans. Roy. Soc. 182. 267.

32. Funke, Physiologie der Hautempfindungen der Physiologie. Hermanns Handbuch. 3. (2). 294.

33. Gad, Ges. Abh. 1. 397.

34. Goldscheider, Die spezifische Energie der Gefühlsnerven der Haut. Monatsschr. f. piakt. Dermatol. 1884. 3. 283.

35. Derselbe, Gesamm. Abhandl. Leipzig 1898.

36. Derselbe, Gesamm. Ahhandl. 1. 199.

36a. Dersel be, Gesamm. Abhandl. 1. 118.

37. Grasset et Vedel, Nouv. Montp. méd. p. 236.

38. Hea d, Pain in visceral Disease. Brain 1893. Pt. 1. 1.; Brain 1894. 2. 339; Brain 1896 3. 153 .

39. Head and Campbell, The Pathology of Herpes Zoster and its bearing on Sensory Localisation. Brain 1900. 3. 353.

40. Head and Rivers, A human Experiment in Nerve division. Brain 1908. 31.

41. Head, Rivers and Sherren, The afferent Nerv. System from a new Aspect. Brain 1905. 28. 99.

42. Dieselben, The Consequences of Injury to the periph. Nerves in Man. Brain 1905. 28. 116.

43. Head and Thompson, The Grouping of the Afferent Impulses within the Spinal Cord. Brain 1906. 116. 1.

44. Herzen, Über die Spaltung des Temperatursinnes in zwei gesonderte Sinne. Pflügers Arch. 1886. 38. 93.

45. B $\mathrm{olm}$, Die Daner der Temperaturempfindungen bei konstanter Reiztemperatur. Skand. Arch. f. Physiol. 1903. 14. 242.

46. Ko $\mathrm{cher}$, Mitteilungen ans den Grenzgebieten der Medizin and Chirurgie. 1896. Heft 4.

47. Kies on, Wandts philosophische Studien. 1898. 14. 586.

48. Derselbe, Wu a tos philosophische Studien 1895. 11. 185.

49. v. Kries and A uerbach, Die Zeitdauer einfachster psychischer Vorgänge. Archiv f. Anat. u. Physiol. (Phys. Abt.) 1877. 297.

50. Kohnstamm, Zur Theorie des Reflexes von hinter-Wurzel auf hintere Wurzel. Zentralbl. f. Physiol. 1900. Heft 18 .

51. Langley, Effect on Nail Growth and Sensation of Section of a Cutan. Nerve. (Proc. Physiol. Soc. 1908. Jan. 25. p. 45.) Journ. Physiol. 1907-08. 36.

52. Lehmann, Die Hauptgesetze des menschlichen Gefühllebens. 1892. 35.

53. Lennander, Mitteil. aus d. Grenzgeb. d. Med. u. Chir. 1902. 10.

54. Derselbe, Obs. on the Sensibility of the abdom. Cavity; translated by A. E. Barker, London 1908. 
55. Lennander, Über lokale Anästhesie und Sensibilität in Organ und Gewebe. Mitteil. aus d. Grenzgeb. d. Med. u. Chir. 15.

56. Derselbe, Weitere Beobachtungen über Sensibilität in Organ und Gewebe. Deutsche Zeitschr. f. Chir 1904. 73. 297.

57. Lewandowski, Die Funktion des zentralen Nervensystems. 1907.

58. Macd onald, L'intermédiaire des Biologistes. 1898. 13. 288.

59. Mackenzie, Contribution to the study of sensory symptoms assoc. with visceral dis. Med. Chron. 1892. 16. 293.

60. Derselbe, Pain. Brain 1902. 25. 368.

61. Marshall, Philos. Review. 1892 and 1895.

62. May, Page, The afferent path. Brain 1906. 56. 758.

63. Derselbe, Investigations into the segment. represent. of movements. Phil. Trans. Roy. Soe. 1897.

64. Mirallie, Ch., Les Radiculites. Le progrès médical. 1908. 37. 13.

65. v. Monakow, Gehirnpathologie. 1905.

66. Müller, Über die Empfindung in unseren inneren Organen. Mitteil. aus d. Grenzgeb. d. Med. u. Chir. 1908. 18. 600.

67. Münzer und Wiener, Beitr. zur Anatomie und Physiologie d. Zentralnerv. Areh. f. Exp. Path. u. Pharm. 35.

68. Nagel, Die Sensibilität der Konjunktiva und Kornea. Pflügers Arch. 1895. 69. 576.

69. Derselbe, Zeitschr. f. Psych. u. Physiol. d. Sinnesorgan. 1869. 10. 277.

70. Neumann, Beiträge zur Klinik des Wärmesinnes. Deutsch. Arch. f. klin. Med. 1903. 76. 106.

71. Nicholls, Physiol. Review. 1895 and 1896.

72. Nothnagel, Beiträge zur Physiologie und Pathologie des Temperatursinns. Deutsch. Arch. f. klin. Med. 1ช67. 2. 284.

73. Pechlin, Jo. Nicol. Pechlini. Observationum physico-medicarum libri tres. Hamburgi. 1691. Liber tertius observat. 9. 410.

74. Petren, Ein Beitrag zur Frage vom Verlauf der Bahnen der Hautsinne im Rückenmark. Skand. Arch. f. Physiol. 1902. 13. 9.

75. Petren und Bergmark, Über Sensibilitätsstörungen bei und nach Herpes Zoster. Zeitschr. f. klin. Med. 68.

76. Petren und Carlstrom, Untersuchungen über die Art der bei Organerkrankungen vorkommenden Reflex-Hyperästhesien. Deutsche Zeitschrift f. Nervenheilkunde. 1904. 27. 465 .

77. Richet, Leipziger Abhandl. 1896. 261.

78. Rendu, Revue de Méd. 1886. 787.

79. Rosenblam, Zeitschr. f. Psych. u. Physiol. d. Sinnesorgan. 1899. 21. 174.

80. Rothmann, Über die Leitung der sensit. im Rückenmark. Berlin. klin. Wochenschr. 190j. Nr. 2 u. 3. Schäfers Textbook 1900.

81. Schlesinger, Über spinale Schweisse und Schweissbahnen. Münch. med. Wochenschr. 1900. 48. 405 .

82. Sherren, Injuries of Nerves and their Treatment. 1908.

83. Sherrington, Exps, in Exam. of the periph. Distribution of the Post. Roots of some Nerves. Phll. Trans. Roy. Soc. 1893. 641.

84. Derselbe, Exps. in Exam. of Periph. Distribution of the Fibres of the Post. Roots of some spinal Nerves. Phil. Trans. Roy. Soc. B. 1898. 190. 45.

85. Dersellbe, The spinal Animal. R. Med. Chir. Trans. 1899. 82. 449.

86. Derselbe, The Spinal Roots and Dissociative Anaesthesia. Journ. Physiol. 1901. 27. 360 and 370 .

87. Dersel be, The Integrative Action of the Nervous System. 1906.

88. Derselbe, Common sensation. Schäfers Textbook of Physiol. 2. 997.

89. Singer und M ünzer, Zehnter Kongress f. inn. Med. 1891. 
90. Sommer, Über die Zahl der Temperaturpunkte der äusseren Haut. Sitzungsber. d. phys.-med. Ges. zu Würzburg. 1900 (1201). 63.

91. Starr, Allen, Local Anaesthesia as a Guide to the Diaguosis of Lesions of the spinal Cord. Amer. Journ. of the Med. Sci. 1892. 104. 15. also Brain 1894. 17. 481.

92. Stransky, Sensibilitätsuntersuchungen an transplantierten Hautstücken. Wiener klin. Wochenschr. 1899, 833 .

93. Strong, Psychol. Rev. 1895 and 1896.

94. Strüm pell, Zur Kasuistik der apoplektischen Bulbärlähmungen. Deutsch. Arch. f. klin. Med. 1881. 28.

95. Tanzi, Sulle sensazioni del fredde e del caldo. Milano-Torino. 1886.

96. Thorburn.

97. 'Thu nberg, Nagels Handbuch d. Physiol. d. Menschen. 1905. 3. 657.

98. Derselbe, Untersuchungen über die Tiefenlage der Nervenenden ete. Skand. Areh. f. Physiol. 1901. 11. 394, 395, 418.

99. Tigerstedt, Studien uber mechanische Nervenreizung. Helsingfors 1880. 66 .

100. Veress, Beiträge zur Kenntnis der Topographie der Wärmeempfindlichkeit. Pflügers Areh. 1902. 89. 33.

101. Derselbe, P flügers Arch. 1902. 89. 1.

102. Vintschgau und Durig, Zeitmessende Versuche über die Unterscheidung zweier elektrischer Hautreize. Pflügers Arch. 1898, 69. 377.

103. Weber, Annotationes anatomicae et physiologicae Lipsiae. 1834.

104. We ber, E. H., Wagners Handb. 3. 2. Abteil. 549.

105. Derselbe, Wagners Handb. III, 2. Abteil. 553.

106. Witmer, The psychological analysis and physical basis of pleasure and pain. Journ. of neur. and mental diseases. 1894. XIX. 209.

Den Fragen über die Verteilung und Funktion der sensorischen Nerven sind endlose Untersuchungen und Beobachtungen von einer Reihe von ausgezeichneten Forschern in der physiologischen, chemischen und anatomischen Welt gewidmet worden. Obgleich wir viel gelernt haben, sind wir noch weit entfernt von unserem Ziel, aber unsere Kenntnis des Gegenstandes hat in den letzten Jahren grosse Fortschritte gemacht.

Die an den niederen Tieren ausgeführten Untersuchungen haben die Grundlage gelegt und den Weg zur Erforschung des Gegenstandes beim Menschen geebnet. Auf diese Weise erhaltene zweifellose Resultate haben unsere Kenntnis rasch vorwärts gebracht und Irrtümer verbessert. Aber das Studium der Empfindung bei niederen Tieren stösst auf viele und unüberwindliche Schwierigkeiten, selbst in einem viel grösseren Umfange, als wie sie bei Problemen geboten werden, welche die motorischen oder efferenten Bahnen betreffen. Um damit anzufangen, ist es unmöglich, wie v. Monakow (65) so scharfsinnig betont hat, eine Antwort bei irgend einem Tiere, ausser beim Menschen, auf die Fragen "ob" und "wie" zu erhalten; „ob" er einen Reiz empfindet und „wie" (Intensität, Modalität, Spezifität, Zeit, Lokalisation usw.). Daher muss das Erkennen des Resultates der Applikation 
irgend einer Art des Reizes bei einem niederen Tiere von dem Forscher auf der Beobachtung irgend eines Reflexes oder einer willkürlichen Bewegung basiert sein. Dies führt notwendigerweise zu der Berücksichtigung von neuen Komplikationen, beispielsweise des Zustandes der Erregbarkeit der verschiedenen Reflexe, Zentren und Bahnen, weiche bei der Bewegung beteiligt sind. Ferner können bei einer Reizung eines sensorischen Nervens nicht nur gewöhnliche Muskelbewegungen hervorgerufen werden, sondern viele andere Reflexwirkungen, wie z. B. Veränderungen in der Stärke und im Rhythmus des Herzens, Zunahme oder Abnahme im Blutdruck durch die gefässverengenden oder gefässerweiternden Nerven und viele andere vasomotorische und sekretorische Vorgänge. Eines oder das andere von diesen kann dann seinerseits der direkte Faktor beim Auslösen einer notorischen Reaktion auf Erregung eines sensorischen Nervens sein, selbst in Fällen, bei welchen der periphere sensorische Nervenmechanismus aus sich selbst (aus verschiedenen unten angegebenen (Gründen) nicht imstande ist, eine richtige spezifische Reaktion oder überhaupt irgend eine wahre sensorische Reaktion zu geben. So kann bei der Untersuchung von einer der einfachsten Formen des Gefühles, wie bei dem Schmerz, ein Tier, selbst wenn es nicht in einem ängstlichen oder aufgeregten Zustand ist, Zeichen der Wahrnehmung oder des Widerstandes zeigen, die leicht für Schmerzäusserungen als Reaktion auf irgendeinen Reiz, sei nun Schmerz vorhanden oder nicht, erklärt werden können. Die Gefahr des Irrtums aus solchen Fehlerquellen, wird, obgleich sie nie fehlt, durch die Benutzung von absolut zahmen Tieren in gutem Ernährungszustand sehr vermindert. Es ist jedoch klar, dass das Problem der Enpfindung bei Versuchen an Tieren nur sehr unvollständig zugänglich ist. Seine vollständige Lösung kann nur durch Untersuchung bei Menschen, sei es im gesunden oder sei es im kranken (zufällig oder freiwillig zugezogenem Zustand) erreicht werden, wo die Aufmerksamkeit einer instruierten menschlichen Person in Anspruch genommen und dieselbe durch den Untersucher mündlich ausgefragt werden kann.

Bei dem Studium der Empfindung ist unsere Kenntnis durch zwei wichtige Faktoren verzögert und verwirrt worden. 1. Durch die mangelnde Strenge der benützten Sprache und ihren ungenauen Gebrauch; 2. durch die fehlerhaften und zweideutigen Untersuchungsmethoden.

Die Sprache ist offenbar eines der Hauptinstrumente oder Hilfsmittel des Gedankens und jede Unvollkommenheit in Instrument oder in der Art es zu benutzen, ist unleugbar verantwortlich für die Verwirrung, hemmt den Prozess und zerstört alle Grundlagen des Vertrauens in das Ergebnis. Es wäre leicht, viele Beispiele zu geben, aber ein oder zwei Beispiele werden genügen, um zu zeigen, wie häufig die bei der Beschreibung der verschiedenen Formen der Empfindung oder des Fehlens der Empfindung benutzte technische Sprache vollkommen irrige Eindrücke ergeben hat. Z. B. wird der Ausdruck 
„Anästhesie" der "offenbar Mangel an Gefühl oder Empfindung bedeutet, häufig in seiner wahren Bedeutung und von vielen Autoren in seiner partiellen Bedeutung angewandt und von einzelnen Klinikern manchmal in einem Sinne und manchmal in dem anderen. Wenn man von einer Hand sagt, sie sei anästhetisch, solite es bedeuten (ausser wenn der Ausdruck anasthetisch als anästhetisch für Schmerz, Wärme, Kälte usw. qualifiziert worden ist), dass die Hand jeder Empfindungsform bar ist. Es hat jedoch häufig der unqualifizierte Ausdruck , anästhetisch" für diejenigen, die ihn anwenden, die Bedeutung, die Vorstellung der Unempfindlichkeit auf Berührung, jedoch der Empfindlichkeit für jede andere Form der Empfindung, zu erwecken. Ohne Bearbeitung des Gegenstandes ist es klar, dass eine solche Fehlerhaftigkeit im Gebrauch eines vollkommen klaren und bestimmten Ausdrucks zu Verwirrung führen muss. Um die Schwierigkeit zu erhöhen, werden wir später sehen, dass durch fehlerbafte Methoden der Tastsinn häufig als vorhanden beschrieben worden ist, wenn er ganz gefehlt hat, und als feblend, wenn er vorhanden war. Überdies sind die gewöhnlich gebrauchten Ausdrücke Hyperästhesie und Hypästhesie von verschiedenen Aatoren gebraucht worden, um einmal eine Steigerung (oder Abnahme) aller Empfindungsarten (Berührung, Schmerz, Wärme, Kälte, Druck, Lage usw.), ein ander Mal nur Steigerung (oder Abnahme) für Tasteindrücke zu bezeichnen. Auch der Ausdruck ,Wärme"-Anästhesie bedeutet tatsächlich nur Verlust der Wärmeempfindung, es könnten jedoch zahllose Beispiele aus der allerletzten medizinischen Literatur angeführt werden, in welchen der Ausdruck im Sinne der Temperaturanästhesie, nämlich Verlust der Empfindung sowohl für Kälte wie für Wärme, gebraucht worden ist. Ähnlich ist es mit den Ausdrücken thermale Hyper- oder Hypästhesie. Es ist nicht nötig; weitere Beispiele zu geben, aber es würde schwer sein, sich etwas vorzustellen, das geeigneter wäre, unsere Ideen eines ohnehin unendlich komplexen Gegenstandes zu verwirren, als Ausdrücke zu gebrauchen, die in einem Augenblick in ibrer wahren und offenkundigen Bedeutung und in einem anderen, in einem verschiedenen oder diametral entgegengesetzten Sinne angewendet werden.

\section{Methoden.}

Für eine genaue Übersicht über die verschiedenen Methoden und Instrumente, die mit Vorteil bei der Untersuchung der Empfindung angewandt wurden, kann auf die wohlbekannten Handbücher und Mitteilungen über den Gegenstand hingewiesen werden, aber einige allgemeine Bemerkungen werden nicht unangebracht sein. Zunächst sind der Zustand des Beobachters und des Beobachteten von grösster Wichtigkeit. Je mehr man die normale oder anormale Empfindung, selbst bei klugen und willigen Personen prüft, 
um so mehr realisiert man beim Forscher die Notwendigkeit einer besonderen Geschicklichkeit und geübten Erfahrung, um die zahllosen Falistricke, die sich auf dem Wege finden, zu vermeiden und verlässliche und richtige Resultate zu erhalten. Überdies muss der Prüfung gewöhnlich Zeit und Geduld reichlich und wiederholt gewidmet werden. Aus Mangel an einem oder mehreren dieser Faktoren sind häufig Resultate erhalten und zur weiteren Verwirung eines bereits hinreichend komplizienten Gegenstandes aufgeschrieben worden.

In bezug auf die beobachtete Person sind die Resultate häufig schlimmer als nutzlos, wenn der Patient nicht frei von Ermüdung, sein geistiger Zustand befriedigend und seine Aufmerksamkeit ungeteilt ist. Die Untersuchungsperioden sollten kurz, obgleich möglichst häufig sein, und die ungeheure Bedeutung der Umgebung und der begleitenden Bedingungen kann durch die Angabe von Head und Thompson (5) hervorgerufen werden, dass „die Genauigkeit, mit welcher Antworten in bezug auf Teile der verminderten Sensibilität gegeben werden, im Winter geringer ist als im Sommer". Dies ist nicht nur Sache der äusseren Temperatur, denn es ist gefunden worden, dass ein feuchter, trüber und nebliger Tag besonders ungünstig zum Prüfen der Feinheiten der Empfindung war. Alles was ein Gefühl von Kälte, ein Frösteln oder einen Anschein von Gänsehaut erzeugt, vermindert stark die Genauigkeit der auf die meisten Empfindungsprüfungen gegebenen Antworten. Die günstigsten Bedingungen sind ein warmer Frühsommertag oder ein heller kalter Wintermorgen in einem gut gewärmten Zimmer. Ferner ist es notwendig, dass zu der Zeit des Prüfens der Patient frei von Unterleibsbeschwerden ist.

Eine allgemeine Bemerkung oder zwei in bezug auf die von den verschiedenen Autoritäten vertretenen und ausgeübten Untersuchungsmethoden, dürften nicht unangebracht sein, obgleich einzelne Einzelheiten notwendigerweise später im Text gegeben werden.

Oberflächliche Berührung wird bequem mit einem kleinen Büschel Baumwolle geprüft; es muss jedoch Sorge getragen werden, dass es nicht steif genug ist, um Druckgefühl (eine sehr gewöhnlich grosse Fehlerquelle beim Prüfen der Empfindung) hervorzurufen, und doch steif genug, um wirksam zu sein, wenn er solchen verdickten Teilen der Haut, wie einer hornigen flachen Hand oder Sohle appliziert wird. Unter gewissen Bedingungen ist es notwendig (wie unten gezeigt wird) die Haut vor dem Versuch für Hauttastsensibilität zu rasieren, aber in vielen Fällen können Schwierigkeiten vermieden werden, indem man v. Freys sinnreiche Einrichtung der Reizhaare, gleichgültig ob die Haut rasiert ist oder nicht, benutzt.

Die Fähigkeit, die gleichzeitige Applikation von zwei Punkten zu unterscheiden, kann leicht geprüft werden, indem man einen Zirkel mit abgerundeten Spitzen benützt. Um genaue Ergebnisse zu erhalten, ist es vorteil- 
haft, die eine oder beide Spitzen (deren intermediäre Distanz nach Belieben bis zu einem bekannten Umfang verändert werden kann) in einer etwas unregelmässigen Anordnung zu applizieren, so dass jede schliesslich gleich oft appliziert worden ist. (Sagen wir zehnmal). Die auf die Reizung gegebene Antwort kann bequem, wie Mc. Dougall vorgeschlagen hat, in Form eines Bruches aufgeschrieben werden; der Zähler schreibt die Art der Antwort auf für eine Stelle und der Nenner diejenige für zwei Stellen und zwar auf folgende Weise: wenn bei einer Entfernung von $4 \mathrm{~cm}$ zwischen den beiden Punkten 3 richtige und 7 falsche Antworten erhalten wurden und die Antworten auf die Applikation eines Punktes $10 \mathrm{mal}$ richtig waren, würde so aufgeschrieben werden:

$$
4 \mathrm{~cm} \frac{1}{2} \mid \frac{10 \mathrm{r}}{3 \mathrm{r} \mathrm{7f}}
$$

oder wie Head und Rivers ihre Resultate durch ähnliches Aufschreiben in Serien von jeder erhaltenen Antwort verzeichneten, nämlich:

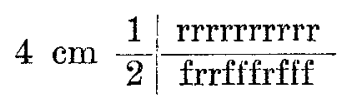

Um Schmerz zu prüfen ist eine einfache Nadel alles was erforderlich ist, aber ein guter Algesimeter oder v. Freys Schmerzhaare leisten in bestimmten Fällen grosse Dienste.

Um die Lokalisation zu untersuchen, ist es nützlich, dem Patienten die Augen zu verbinden oder noch besser, irgend einen Schirm zwischen das Auge und den zu prüfenden Teil zu bringen. Um die Lage der Lokalisation, wie sie von dem Patienten angegeben wird, zu bezeichnen, sind von Head und Rivers verschiedene Methoden angewandt worden, nämlich Beschreibung der Lage des Fleckens, indem auf den angegebenen Flecken hingewiesen wird, und für einige Beobachtungen Viktor Hen ris Methode den gereizten und bezeichneten Flecken auf einer lebensgrossen Photographie des untersuchten Teiles anzugeben.

Um die Wärmeempfindung zu prüfen, genügen im allgemeinen die gewöhnlichen einfachen Methoden, aber wo es wünschenswert ist, den Druck, welcher gewöhnlich die Applikation begleitet und die daraus resultierende Reaktion der tiefen Sensibilitat zu vermeiden, hat Sherrington eine Dunkelwärmeprobe benützt. Die Wärmeausstrablung aus einer starken Quelle, z. B. einer Bogenlampe wurde durch eine dünnwandige Hohlglaslinse, die mit Schwefelkohlenstoff ausgefüllt war, welches hinreichend Jod enthielt, um sie für Licht undurchsichtig zu machen, gesammelt. Dank der Güte von Professor Sherrington, war es dem Verfasser gestattet, den selben Apparat zu benützen, als er die Empfindung bei einem Schimpansen untersuchte, und er hat ähnliche Kunstgriffe in vielen Fällen beim Affen benutzt. Ein Nachteil seiner Anwendung ist der, dass die tatsächlich appli- 
zierte Temperatur unbekannt ist und dass sie nicht selten höher ist, als man wünscht und dass sie auf diese Weise unerwünscht andere spezifische Reaktionen hervorruft.

Weitere Einzelheiten werden im Laufe dieses Essays vorkommen, aber zwei Punkte treten klar in der bahnbrechenden Arbeit von Head, Rivers, Sherren und Thompson hervor, deren auffallende Ergebnisse die Grundlage dieses Essays bilden, nämlich: 1. Die von ihnen angenommenen sehr einfachen Untersuchungsmethoden. 2. Dass sie in klinischen Fällen, um bestimmte und unzweideutige Resultate zu erhalten, die Schwelle soviel als möglich vermieden und in einem weiten Abstande über und unter diesem Punkte prüften.

Angesichts der oben entwickelten. Tatsachen ist es klar, dass die Literatur dieses komplexen Gegenstandes (sensorische Nerven und periphere Sensibilität), die sich auf ein weites verwirrendes Labyrinth gedruckter Bände beläuft, zahllose Irrtümer verzeichnet, welche hauptsächlich aus ein oder zwei Quellen entstanden sind; erstens aus Unvollkommenheiten in der Terminologie des Gegenstandes oder in der Art und Weise sie anzuwenden; zweitens noch mehr aus den gewöhnlich angenommenen ungenauen Untersuchungsmethoden, welche zahllose irrige Ergebnisse und Resultate ergeben haben. So oft ist dies der Fall, dass es bei manchen Beispielen viel besser gewesen wäre, wenn die erhaltenen Resultate nie aufgeschrieben worden wären. Unter diesen Umständen ist es augenblicklich viel vorteilhafter, anstatt im einzelnen oder historisch die Arbeit der früheren Forscher zu betrachten, reinen Tisch zu machen und im einzelnen die Flut des neuen Lichtes zu betrachten, das von einzelnen Forschern kürzlich auf diesen Gegenstand geworfen worden ist und das einen vollkommen neuen Standpunkt bedingt, von dem aus man die ganze Frage ansehen kann.

In einer Mitteilung über die Beurteilung der afferenten Bahn im Gehirn (Sommer 1906, p. 743) ist von dem Verfasser dieser Monographie behauptet worden, dass gewisse Forscher, wie Head und Sherren und Head, Rivors und Sherren nicht nur eine neue Seite, sondern ein vollkommen. neues Kapitel in der neurologischen Wissenschaft angefangen hatten. Seit dieser Zeit haben Head und Thompson und Head und Rivers sich in ihrer Wissenschaft eine neue Dankesschuld erworben. Es wäre gut, von einer Betrachtung einzelner dieser Resultate der obigen Forscher auszugehen, welche sich auf die hier in Betracht kommenden Fragen beziehen. Zuuächst waren ihre Ergebnisse nicht nur auf Beobachtungen von zahlreichen klinischen Fällen, die sich über viele Jahre erstreckten, basiert, sondern es wurden auch diese im Lichte der neuen Resultate untersucht, welche sie durch Durchschneidung der präaxialen Gruppe von afferenten. Nerven des Vorderarmes an einem von ihnen erhielten (nämlich die radialen Hautnerven und die äusseren Hautnerven). Der einzig dastehende, auf diese Weise er- 
haltene Vorteil war, dass der zu untersuchende Patient Dr. Head selbst ein geschulter Beobachter war. Hierzu kommt, dass der genaue sensorische Zustand des Armes und der Hand vorher erhalten wurde, die Verletzung genau der Zeit und dem Umfang nach bekannt waren und die unter den günstigsten Bedingungen erhältlichen Resultate von Tag zu Tag und von Jahr zu Jahr von einem besonders geschickten und geschulten Forscher (Dr. Rivers) beobachtet wurden. Frei von den kleinen Mühen eines geschäftigen Lebens, mit den Hilfsquellen der modernen Wissenschaft bewaffinet, erforschten Head und Rivers in kleinsten Einzelheiten, besonders an jedem Ende der Woche, die Veränderungen, die an dem affizierten Teile währeud eines Zeitraumes von mehr als vier Jahren auftraten.

\section{Anmerkung:}

Einige der erhaltenen Resultate zusammen mit denjenigen einer Untersuchung zahlreicher ausgewählter Krankheitsfälle, welche den Gegenstand betreffen, sind in folgenden Mitteilungen veröffentlicht worden: The afferent system from a new aspect. Von Head, Rivers and Sherren. Brain 1905, Vol. XXVIII.

Injury to the peripheral werves in man. Von Head and Sherren. Brain 1905, Vol. XXVIII.

The grouping of afferent impulses within the spinal cord. Von Head and Thompson. Brain 1906, Part. CXVI.

Eine vollständigere und detailliertere Übersicht der Untersuchung wird in kurzem von Heads Hand im Brain, a human experiment in nerve division, 1908, Vol. XXXI betitelt, erscheinen und ich möchte meinen Freunden Head und Rivers meine Dankbarkeit aussprechen, dass sie mir in ihre Korrekturbogen (soweit sie fertig waren) vor ihrer Veröffentlichung Einblick gewährt haben.

Einzelheiten kann man in den Originalen finden, aber einige von diesen Forschern erhaltenen wichtige Punkte werden im Umriss in den folgenden Zeilen betrachtet werden, denn sie fügen nicht nur grosse und bedeutende Kenntnis zu dem Problem der Empfindung hinzu, sondern sie verhelfen dazu viele der scheinbaren Widersprüche in den Berichten über frühere Untersuchungen des Gegenstandes zu erklären.

\section{Disensibilisierte Hant.}

Als ein Ergebnis der Durchschneidung der radialen und äusseren Haut. nerven in Heads Arm wurde gefunden, dass Verlust jeder Art von Hautsensibilität über ein ausgedehntes Areal. in der radialen Hälfte des Vorderarms und des Handrückens hinaus eintrat. Reizung mit Baumwolle, der Spitze einer Nadel, die Applikation jeder Form von Wärme und Kälte 
blieben unbemerkt und die beiden Spitzen des Zirkels konnten nicht unterschieden werden. Wenn jedoch derselbe Teil mit der Spitze eines Bleistiftes, dem Kopf einer Nadel oder selbst mit dem Fingerballen berührt wudde, wurde der Reiz sofort bemerkt und mit bemerkenswerter Genauigkeit lokalisiert. Daher wurde die bemerkenswerte Tatsache sofort konstatiert, dass Zerstörung aller sensorischer Nerven nach der Haut den Teil auf die meisten jener Reize reagierend lässt, welche gewöhnlich vom Arzt und Chirurgen als ein Prüfstein der Sensibilität auf Berührung benutzt werden. Auf einem solchen Areal können alle Arten des Druckes, wie Berührung mit einem Bleistift oder den Federn eines Federkiels bemerkt und mit beträchtlicher Genauigkeit lokalisiert werden. Durch gewöhnliche Methoden wird Reizung für Schmerz, Wärme, Kälte, Berührung usw. selten ohne irgend einen Druck ausgeübl, daher muss sie, damit der Reiz eine richtige spezifische Reaktion auslösen kann, von einer so spezifischen Art sein, dass das Druckelement in der Empfindung im Hintergrund bleibt. So kann ein Nadelstich der Haut bemerkt und lokalisiert werden, aber wenn er nicht als "Schmerz" verursachend erkannt wird, kann das Erkennen der Reizung vollkommen von der tiefen Sensibilität, nämlich von der Wirkung der afferenten Fasern, welche eutlang der motorischen Fasern und Sehnen laufen, herrühren, deren Existenz Sherrington (2) zuerst nachgewiesen hat. Dass ein Stich mit einer Nadel gefühlt und gut lokalisiert wurde, ist keinerlei Beweis für das Vorhandensein einer Hautempfindlichkeit für Schmerz.

Überdies bot dieses Areal in Heads Vorderarm und Hand, welches daher nur von afferenten Fasern des Muskelnerven innerviert wurde, eine fast einzige Gelegenheit zur Untersuchung der sogenannten ,tiefen Sensibilität" 1). Sherrington wies nicht nur afferente Fasern in Muskelnerven nach, sondern er verfolgte sie vermittelst der Degenerations- und Erregungsmethoden in einer Richtung in den Muskeln und in der anderen im Rückenmark, und er zeigte, dass sie in letzteres durch die hinteren Wurzeln von jenen Segmenten eintraten, an welchen die motorischen Nervenfasern für dieselben Muskeln entspringen. Diese afferenten Nerven lieferten die anatomische Grundlage für den Muskelsinn, aber, wie besonders Head und Sherren gezeigt haben, dienen sie nicht nur dem Vermögen Bewegung zu bewerten.

Welche Flut von licht $\mathrm{Heads}$ neue Methode sofort auf diesen Gegenstand wirft, wird aus folgender Betrachtung klar.

\section{Tiefe Sensibilität.}

Die Erzeugung einer Empfindung durch die Applikation einer Kamelhaarbürste oder einem Wischer von Baumwolle auf $\mathrm{He}$ a ds entnervtes Haut-

1) Roux und Parisot, auch Déjerine und Egger (loc. cit.p. 3) und viele andere haben Fälle von Dissoziation der Haut durch tiefe Sensibilităt beschrieben. 
areal hing grossenteils von dem gleichzeitigen Druck und der Schnelligkeit der Berührung ab. Je allmählicher die Applikation und je kleiner der Druck, umso seltener gab es ein Empfindungsergebnis. Wenn sie plötzlich und vertikal zar Haut appliziert wurde, so dass ein Delle verursucht wird, wurde eine leise Berührungsempfindung (Druckberührung) erzeugt. So wurde Druck, selbst leiser Druck oder irgend eine Delle auf der Haut schnell bemerkt und mit bemerkenswerter Genauigkeit lokalisiert. Ein Druck wurde jedoch nicht wahrgenommen, wenn er der affizierten Haut, die von den subkutanen Strukturen, um eine Falte zu bilden, in die Höbe gehoben wurde, appliziert wurde. Letzteres zeigte, dass die Druckempfindlichkeit nicht von Nerven herrührte, die noch in der Haut nach der Operation blieben. Übermässiger Druck im obigen Areal erzengte heftigen Schmerz und mit Cattells Algimeter wurde gefunden, dass Schmerz selbst mit kleinerem Druck als auf der normalen Seite ausgelöst werden konnte.

\section{Anmerkung:}

In Déjérine und Eggers Fall (loc. cit. S. 4) von totaler Hautanästbesie mit andauernder tiefer Sensibilität waren die Muskeln auf Druck auch schmerzhaft, obgleich die relative Druckmenge, die erforderlich ist, um Schmerz auf der normalen und der angegriffenen Seite zu erzeugen, nicht angegeben ist. In einem späteron und ähnlichen Fall von Madame Déjérine (loc. cit. S. 639) bestand, obgleich der Arm kalt, zyanotisch und ödematös war, vollkounmenes Erkennen des Druckes, der Lage und der Knochenempfindlichkeit trotz totaler. Unempfindlichkeit auf Berührung, Schmerz und Temperatur. Auf Druckschmerz haben auch Camus und Sézary, S. 537, hingewiesen.

Die Eigenschaft der Rauheit wurde leicht erkannt und indem man Grabam Browns Ästhesiometer benutzte, wurde gezeigt, dass diese Eigenschaft ebenso leicht auf der affizierten, wie auf der nicht affizierten Seite erkannt wurde. Head und Sherren waren imstande zu zeigen, dass vollkommenes Erkennen der passiven Bewegung der Glieder möglich war, wenn nur die Muskelnerven unversehrt waren. Auch selbst die geringste durch den unterbrochenen Strom induzierte Kontraktion des Abductor indicis oder pollicis erzeugte eine deutliche in dem Muskel lokalisierte Bewegungsempfindung. Es wurde kein Schmerz hervorgerufen, wenn nicht der Muskel in Krampf verfiel.

Andererseits blieben in obigem entnervten Hautareal der Stich einer Nadel und der unterbrochene Strom gänzlich umbemerkt. Die affizierten Teile konnten selbst mit Chloräthyl gefroren oder auch verbrannt werden, ohne Schmerz zu erzeugen und Temperaturen von $0^{\circ}$ bis $60^{\circ}$ oder darüber verfehlten vollkommen die Empfindung von Wärme oder Kälte hervorzurufen. Es wurde keine Empfindung erfahren beim Herausziehen eines 
Haares und die Haut wurde bis zu ihrem Maximalumfang in die Höhe gehoben, ehe das Haar herausgezogen wurde, ohne Empfindung zu erzeugen. Ferner waren Applikationen einer leichten Berührung (wie mit einem Baumwollwischer) und die Grösse und die Gestalt der Gegenstände ganz ausserhalb des Bewusstseins und das Erkennen von zwei gleichzeitig applizierten Punkten (Zirkelversuch) fehlte vollkommen.

\section{Anmerkung:}

Es ist schwierig, zuverlässigen Bericht in bezug darauf zu erhalten, wann die Nerven der tiefen Sensibilität nach der Durchschneidung regenerieren, aber bei Head und Sherren ist das volle Erkennen des Druckes und der tiefen Sensibilität sieben Monate nach der Verletzung zurückgekehrt. Von anderen Fällen weiss man, dass tiefe Sensibilität nicht anfängt wiederzukommen, bis einige Zeit nach dem Beginn der Regeneration der protopathischen Sensibilität und lange vor der epikritischen Sensibilität. (Siehe unten.)

Um zusammenzufassen, sieht man, dass die tiefe Sensibilität aus mehreren Komponenten zusammengesetzt ist, nämlich den Impulsen, welche hauptsächlich denjenigen des Druckes, des Widerstandes, der passiven Lage, der aktiven und passiven Bewegung und möglicherweise Verlagerung der Haut unterstehen. Aus den obigen Ergebnissen in dem Versuch von Head sieht man, dass tiefe Sensibilität zum Erkennen und der Lokalisation der Impulse des Druckes, der Druckberührung, des Druckschmerzes, der Rauheit der Haut und der Muskelbewegung dient. Sie bietet keinerlei Hilfe bei dem spezifiscben Erkennen des leichten Druckes, Nadelstiches, Wärme oder Kälte oder beim Zirkelversuch.

In Fällen von Durchschneidung des medianen oder ulnaren Nerven haben alle früheren. Forscher festgestellt, dass die Empfindung gewöhnlich über das ganze, dem verletzten Nerven zugehörige Areal vermindert und nur über einen kleinen Teil ganz verloren ist. Wenn daher der mediane Nerv durchschnitten ist, ist die Empfindung über einen beträchtlichen Teil sowohl des Zeigefingers wie des Mittelfingers vollkommen verloren, während gewöhnlich behauptet wurde, dass in der inneren Handfäche, innerhalb des Areals, von welchem die Anatomen sagen, dass es ron diesem Nerven versorgt wird, die Empfindung vermindert und nicht gänzlich vernichtet wurde. Ähnlich wird bei einem durchschnittenen ulnaren Nerv vollständige Unempindlichkeit des kleinen Fingers und einem variablen Teil der ulnaren Seite der innereu Hand erzeugt, mit partiellem Verlust der Empfindung über ein grosses Areal der Handfläche und der ulnaren Hälfte des Riugfingers. Von dem Areal, in welchem die Empfindung nur teilweise verloren wird, wurde früher eine doppelte Versorgung von Nerven angenommen, mit anderen Worten greifen aneinander grenzende Nerven über ihr Gebiet in das be- 
nachbarte über. Wenn dem so ist, ist es klar, dass Zerstörung des ulnaren Nerven eine Verminderung der Sensibilität über den medianen Teil der Handfläche verursachen muss; und dieser Verlust sollte genau im Verhältnis zu der Empindungsmenge, die bleibt, variieren. Aber sorgfältige und genaue Prüfung der Hand entdeckt nicht die geringste Verminderung der Empfindung über der medianen Hälfte der Handfäche, infolge von Durchschneidung des ulnaren Nerven. Das was die verminderte Sensibilität, die gewöhnlich in den Rändern eines durch Durchschneidung eines peripheren Nervens begrenzten Areals vorkommt, genannt worden ist, ist in Wirklichkeit ein Zustand, in welchem manche Arten der Sensibilität verloren und audere erhalten sind.

Head hat gezeigt, dass innerhalb eines solchen Hautareals (die intermediäre Zone, wie sie oft genannt wird) alle Empfindung für leichte Berührung, das Erkennen der Zirkelspitzen, das Erkennen niederer Temperaturgrade, sagen wir zwischen $22^{\circ}$ und $40^{\circ} \mathrm{C}$, und das Vermögen der genauen Lokalisation alle vollkommen vernichtet sind, während im selben Areal der Stich einer Nadel sofort gefühlt und ziemlich gut lokalisiert wird, während extreme Wärme oder Kälte, sowie Eis oder eine Temperatur unter $20^{\circ} \mathrm{C}$ und Wärme über $40^{\circ} \mathrm{C}$ gut erkannt werden.

Bei Heads eigener Untersucbung an der vorderen Oberfläche des Vorderarms entsprach der Verlust der Empfindung auf Stich und Baumwolle genau einander, weniger nach der radialen Gegend zu. Aber auf dem Handrücken existierte ein schmaler Streifen $(2 \mathrm{~mm}$ breit), der unemptindlich für Baumwolle, aber empfindlich für Stiche war. Die durch letztere hervorgerufene Schmerzempfindung war lebhaft und sebr viel unangenehmer, als die an normalen Teilen erzeugte. Wenn an einem Haar im analgesischen Areal gezogen wurde, wurde keine Empfindung erweckt, aber sowie an einem Haar in dem schmalen Streifen, der empfindlich für Stiche aber nicht für Berührung war, gezogen wurde, wurde eine diffuse und ungewöhnlich unangenehme Empfindung erzeugt. Ähnlich ist es dort mit der Applikation eines unterbrochenen Stroms. Es sehien, als ob dieser schmale Streifen, der zweifellos eine grössere Reaktion auf schmerzhafte Reize als normale Teile gab, hyperalgetisch war. Weitere Untersuchung, besonders mit dem Algesimeter, zeigten jedoch, dass er tatsächlich mit geringerer Sensibilität für schmerzhafte Hautreize als normale Teile begabt war; für einen stärkeren Reiz war eine höhere Schwelle notwendig, ehe irgend eine Reaktion für Schmerz erhalten wurde.

\section{Wiedererlangung der Sensibilität in dem Gebiet eines durchschnittenen Hautnerven.}

Wenn ein Hautnerv sorgfältig durchschnitten worden ist, so geschieht die Erholung der Empfindung nicht, wie gewöhnlich geglaubt wird, durch 
eine allmähliche Steigerung bis zu allen Formen der Sensibilität, die in Teilen anfängt, wo die Empfindung nie ganz verloren gewesen ist; sondern das affizierte Areal wird zuerst empfindlich für Stiche, und kurz danach und allmählich für die extremeren Formen der Kälte und Wärme. Erst nach einer weiteren Zwischenzeit von mehreren Monaten beginnen die höheren Formen (z. B. leichte Berübrung, genaue Lokalisation, Unterscheiden der Temperatur und Erkennen der beiden Zirkelspitzen) zurückzukebren. So war in Heads Fall (38), nachdem die radialen und äusseren Hautnerven durchschnitten waren, wovon die Ergebnisse oben berichtet worden sind, keine deutliche Veränderung in der Empfindung des anästhetischen Areals während sieben Wochen bemerkbar; dann (43 Tage nach der Operation) wurde das Areal der Hautanalgesie weniger umfangreich (an der Beugeseite des Vorderarmes um $8 \mathrm{~cm}$ ) und stimmte nicht mehr mit dem der taktilen Anästhesie überein, die, um Wiederholung zu vermeiden, zwölf volle Monate absolut unverändert blieb. Überdies waren die Grenzen der Hautanalgesie nicht mehr bestimmt, sondern aus Inselchen oder Punkten (etwas dumpf) der Empfindung zusammengesetzt, und es kann sein, dass zwischen diesen Inselchen Sensibilität nicht angetroffen wird. Fünfzig Tage nach der Operation wurde gefunden, dass dieses Schrumpfen an Umfang der Analgesie fortdauerte und auch, dass der Verlust der Kälteempfindung annähernd im Umfang dem Verlust der Stichempfindung entsprach; aber dort wo der Teil schwach empfindlich für den letzteren Reiz war, schien die Empfindlichkeit für Kälte zu fehlen, und sechs Tage später (56 im ganzen) ist die Empfindung für Nadelstich in der Endund Basalphalanx des Daumens erschienen. 86 Tage nach der Operation war die Empfindung für Nadelstiche vollständig im Vorderarm, über den ganzen Daumen und über der Haút der ersten Handwurzel vorhanden. Zu dieser Zeit wurde auch die Kälte (von $17^{\circ} \mathrm{C}$ abwärts) zuerst in der Endphalanx des Daumens, und Hitze über $45^{\circ} \mathrm{C}$ erkannt. Zu dieser Zeit erzeugte auch Ziehen der Haare am Vorderarm zum ersten Male eine schmerzhafte unangenehme Empfindung, die oft verschwand und wieder kam ohne weitere Reizung.

112 Tage nach der Operation wurden zum ersten Male Kältepunkte gefunden, nämlich am oberen Vorderarm, und zum erstenmal seit die Erholung der Empfindung begann, konnte behauptet werden, dass jene Teile, welche auf Wärme und Kälte reagierten, es bestimmt und ständig taten. Die Endphalanx des Daumens reagierte zweifellos auf Temperaturen über $45^{\circ}$, und selbst die proximale Phalanx des Daumens war empfindlich für Eis geworden, obgleich sie noch anästhetisch für Wärme war.

137 Tage nach der Operation hatten die Kältepunkte an Zahl zugenommen und der ganze Vorderarm reagierte auf Kälte, aber noch nicht auf Wärme, ausser über $50^{\circ} \mathrm{C}$.

152 Tage nach der Operation wurde der Stich über den ganzen Vorder- 
arm und die Hand hin, ausser auf einem kleinen Fleck auf letzterer, erkannt. Ein Geschwür auf der Handrücken, das zufällig 76 Tage nach der Operation entstand, konnte man nicht heilen, fing aber nun an zu stechen und zeigte Erkennen schmerzhafter Reize. Es heilte dann schnell und dauernd. Über dem ersten $Z$ wischenraum wurden nun zum erstenmal Kältepunkte entdeckt.

161 Tage nach der Operation gab das mit Baumwolle gestreichelte Haar eine besondere Empfindung, obgleich, nachdem die Teile rasiert worden waren, weder Baumwolle noch v. Freys Haare irgend eine Empfindung auslösten.

166 Tage nach der Operation wurde zum ersten Male ein Wärmepunkt entdeckt nnd zwar im oberen Vorderarm. Einige Tage später (173 Tage) war Empfindung auf Nadelstiche über den ganzen Vorderarm und die Hand vorhanden. Die Kältepunkte nahmen schnell zu und in geringerem Umfange auch die Wärmepunkte, während 190 Tage nach der Operation ein Wärmepunkt auf der ersten Phalanx des Daumens entdeckt wurde. Mit der allmählichen Rückkehr der Sensibilität für Schmerz, Kälte und Wärme tendierten die Empfindungen danach weit zu irradieren und sie waren häufig auch auf einen Ort bezogen, der entfernt vom Reizungspunkt war, z. B. von dem proximalen Teil des Vorderarms, auf den Daumen oder vom Knöchel des zweiten Metakarpus auf den Daumen. Aber die Lage dieses Ortes (?), auf den bezogen wurde, war nicht zufällig, sondern konstant. Die eigenartige Sensibilität der Haare, wie oben beschrieben, wurde besonders an der Hand deutlicher, aber das ganze affizierte Areal des Vorderarms und der Hand blieb noch unempfindlich gegen $v$. Freys Tasthaare und gegen leichte Berübrungen von Baumwolle während nochmals 150 Tagen.

364 Tage nach der Operation war das früheste, dass Empfindlichkeit gegen leichte Berührung in einem sauber rasierten Teile bestimmt erkannt wurde, und zwar geschah das am Vorderarm.

470 Tage nach der Operation war es möglich eine dem Vorderarm applizierte Temperatur von $37^{\circ} \mathrm{C}$, und drei Wochen später eine Temperatur von $34^{\circ} \mathrm{C}$ gerade zu bemerken.

Ferner war die Empfindung der Wärme gut lokalisiert, und sie hatte nichts von der diffusen Ausstrahlung und der Tendenz, auf einen anderen Ort bezogen zu werden, die so charakteristisch für die durch Reizung der Hitzepunkte im früheren Stadium der Erholung erzeugte Empfindung ist. Diese Ausstrablung und Diffusion nahmen immer weiter überall ab, und am 13. November 1904, 568 Tage nach der Operation, fing ein Teil des Handrückens an empfindlich gegen Wärme von $35^{\circ} \mathrm{C}$ zu sein. Während des Winters gab es wenig Besserung, tatsächlich war in vielen Einzelheiten die Empfindlichkeit leicht vermindert, aber mit dem Beginn des folgenden Sommers dauerte der weitere Fortschritt an, und ausser über den Knöcheln 
des Zeige- und Mittelfingers war die Empfindlichkeit für Baumwolle nach dem Rasieren für Nr. 5 der Freyschen Tasthaare und für mittlere Temperatur von $24^{\circ}-40^{\circ} \mathrm{C}$ überall vorhanden.

\section{Protopathische Sensibilität.}

Die oben beschriebene mittlere Zone und ein Teil im ersten Stadium der Wiederkehr der Sensibilität sind in ihren negativen Eigenschaften, nämlich in ihrer Unempfindlichkeit für Baumwolle, Temperaturen zwischen $22^{\circ}$ und $40^{\circ}$ und den Zirkelversuch, gleich. Ferner ist der Charakter der Reaktion in jedem Falle ähnlich, denn ein Nadelstich verursacht einen heftigen Schmerz stechender Natur, der ausstrahlt, und indifferent lokalisiert ist. Ähnlicherweise erzeugen die Extreme von Hitze und Kälte (über $40^{\circ} \mathrm{C}$ und unter $20^{\circ} \mathrm{C}$ ) spezifische Empfindungen, welche weit ausstrahlen und stechender Natur sind. Wenn der Teil mit Haar versehen ist, erzeugt Ziehen an einem Haar eine sehr intensive schmerzhafte und unaugenehme Empfindung, die auch weit ausstrahlt, während leichtes Bürsten dieser Haare mit Baumwolle eine Empfindung erzeugen wird, die verschieden von der ist, welche man bei normalen Teilen erhält; denn sie besteht nicht nur aus einem sonderbaren Stechen oder Ameisenkriechen, sondern sie radiiert weit vom Reizungspunkte aus, und wird dazu häufig auf Teile in einer entfernten Gegend bezogen. Doch wird der Teil, anders wie die normale Haut, wenn rasiert, vollkommen unempindlich (leichte Applikation) für Baumwolle. Wenn überdies eine Nadelspitze leicht über die Haut von normalen zu anormalen Teilen gezogen wird, nimmt die Empfindung an Intensität und Lebhaftigkeit $\mathrm{zu}$, sowie die Linie überschritten ist, an welcher leichte Berührung nicht mehr erkannt wird, d. h. die Transitionslinie ist zwischen dem gesunden und dem protopathischen Areal eine scharfe. Obgleich die intermediäre Zone und eine sich erholende Hand einander in ihrem Reaktionsmangel auf zartere Reizformen, ebenso wie in der weiten Ausstrahlung der von ihnen hervorgerufenen Empfindung ähneln, unterscheiden sie sich in einer wichtigen Eigenheit, denn obgleich ihre Sensibilitäten in der Qualität gleich sind, differieren sie im Grade. Von der intermediären Zone kann man, ganz abgesehen von der Fühllosigkeit für leichte Berührung, mit Recht von Abgestumpftheit sprechen, d. h. von einem Areal verminderter Sensibilität, während das mit einer Nadel gestochene, sich erholende Hautareal einen grösseren Schmerz hervorruft als die normale Haut. Ähnlich scheinen in diesem Areal die Extreme von Hitze und Kälte wärmer bez. kälter als in normalen Teilen zu sein; die Empfindungen, die hier entstehen, sind lebhafter als diejenigen von der normalen Haut.

Wenn man die verschiedenen Zeitpunkte berücksichtigt, bei welchen diese verschiedenen Formen der Sensibilität in einem regenerierenden Nerven- 
areal wiederkommen, ersieht man auch, dass mehrere Monate, ehe irgend eine Reaktion auf feinere und zur Unterscheidung führende Formen der Reizung (leichte Berührung, warm oder kühl genannte Temperaturen von $24^{\circ}$ bis $38^{\circ} \mathrm{C}$, feine Lokalisation u. s. w.) erhaltbar ist, die oben erwähnten Eigenschaften der Sensibilitüt (Schmerz, Haarempfindlichkeit, Extreme von Hitze und Kälte, alle von einem weit irradiierenden Charakter) mehr oder weniger „pari passu“ wiederkehren und schnell und kräftig nachweisbar sind.

Dieser Form von Sensibilität, die so charakteristisch für Teile ist, zu denen die Empfindung zurückgekehrt, hat Head den Namen protopathische Sensibilität gegeben.

Wir wollen obige Form der Sensibilität etwas mehr im einzelnen betrachten. Eine Temperatur von $50^{\circ} \mathrm{C}$ oder darüber ist imstande, Schmerz sowohl wie Hitze hervorzurufen, aber der Patient gibt an, dass, obgleich Schmerz und Stechen durch Reize dieser Temperatur hervorgerufen werden, sie von einer bestimmten Empfindung von Hitze begleitet werden. Wenn wiederum ein Reagenzrohr von $45^{\circ} \mathrm{C}$ über die Hand von normalen zu anormalen Teilen geführt wird, scheint es heisser zu werden, sobald wie das protopathische Areal erreicht wird. Dieses selbe Areal ist jedoch vollkommen unempfindlich für Wasser von $35^{\circ} \mathrm{C}$, welches für die normale Hand entschieden warm ist. So reagiert der sich erholende Teil stärker auf eine Temperatur von $45^{\circ} \mathrm{C}$ und weniger stark auf eine andere von $37^{\circ} \mathrm{C}$, was beweist, dass keine vermehrte Empfindlichkeit für Wärme im ganzen vorhanden ist. Ähnliche Phänomene entstehen mit der Kälte, und die durch Temperaturen unter $18^{\circ} \mathrm{C}$ erzeugte Reaktion ist grösser über dem protopathischen als über den normalen Arealen.

Aus den Protokollen über die Wiederkehr der prothopathischen Sensibilität sieht man, dass das Erkennen der Kälte etwas dem der Hitze voranzugehen schien. Ein klares und bestimmtes Erkennen der äussersten Kälte (unter $20^{\circ} \mathrm{C}$ ) war von dem Vorhandensein von einem oder mehreren kalten Flecken begleitet. Ähnlicherweise hing die vollständige und definitive Wiederherstellung der Empfindlichkeit gegen Hitze in einem Teil von dem Wiedererscheinen eines heissen Fleckens oder Flecken ab. Dank der Abwesenheit der Temperaturempfindlichkeit überall ausser in der Nachbarschaft dieser Flecken war die Erforschung ihrer Natur leicht, obgleich eine Schwierigkeit in bezug auf die Bestimmtheit und die Lebbaftigkeit, mit denen die verschiedenen Flecken reagieren, entstand. Unter günstigen Bedingungen reagieren einige Flecken auf jede geeignete Reizung, und Head (38) lernte jeden dieser "erstgradigen" "Flecken erkennen, denn sie. konnten ständig mit Leichtigkeit herausgefunden werden. Eine beträchtliche Anzahl von Flecken reagierte jedoch mit Bestimmtheit erst nach einer Nacht Ruhe, nach Vermeidung der Ermüdung oder wenn sie zuerst gereizt oder wenn der reizende Stab gerade vom Eis entfernt worden war. Solche „zweitgradigen " Flecken 
sind in der normalen Haut vorhanden, aber ihr Erkennen ist in einem protopathischen Areal durch das totale Fehlen von jeder, ausser punktförmiger, Wärme-Empfindlichkeit leicht gemacht.

Die Hitzeflecken zeigen weniger Variationen in der Tätigkeit und fast alle von ihnen reagieren konstant auf geeignete Reize, d. $h$. am allerbesten auf eine Temperatur zwischen $44^{\circ}$ und $43^{\circ} \mathrm{C}$. Eine hohe Temperatur von $55^{\circ} \mathrm{C}$ oder mehr ruft nicht nur die Empfindung von Hitze hervor, sondern später ein sonderbares Stechen, und wenn sie fortgesetzt oder gesteigert wird, wird sie Schmerz erzeugen, der die besondere Wärmeempfindung besitzen kann. Ferner sind die Hitzeflecken viel geringer an Zahl als die Kälteflecken. Die Hitzeflecken reagierten nie auf eine Temperatur unter $37^{\circ} \mathrm{C}$, auch erweckt ein Kälteflecken nicht die Kälteempfindung über $26^{\circ} \mathrm{C}$ und selten über $25^{\circ} \mathrm{C}$. Es ist kaum zu wiederholen notwendig, dass all diese Empfindungen die stechenden und irradiierenden Eigenschaften hatten, die für die protopathische Sensibilität charakteristisch sind.

Ein oder zwei Punkte mögen erwähnt werden. Innerhalb weiter Grenzen würde es scheinen, als ob die Zahl der gereizten Flecke von grösserer Bedeutung für die Schärfe der ausgelösten spezifischen Empfindung ist, als die Intensität der einem Flecken applizierten Kälte. Zwei Gegenstände von gleicher Oberfläche, von denen einer kälter ist als der andere, werden eine kältere Empfindung erzeugen; während ein Gegenstand von, sagen wir, $15^{\circ} \mathrm{C}$, der eine Gruppe von Kälteflecken reizt, kälter erscheinen wird, als ein einem Kälteflecken applizierter eiskalter Stab. Wegen der Zerstreutheit der Wärmeflecken konnte eine ähnliche Untersuchung mit Wärme nicht befriedigend ausgeführt werden.

Ein sonderbares und wohlbekanntes Phänomen ist das der sogenannten "paradoxen Kälte", die auch an der normalen Haut gezeigt werden kann, indem nämlich Reizung eines Kältefleckens in einem von Wärmeflecken freien Areal mit einem Stab von einer Temperatur von über $44^{\circ} \mathrm{C}$ eine lebhafte Kälteempfindung erwecken kann. Keinem ist es bis jetzt unstreitig gelungen, eine analoge Hitzeempfindung durch Reizung eines Wärmefleckens mit Kälte zu erzeugen (Strümpell contra). Ferner gelang es $\mathrm{Head}$ und Rivers nicht, eine spezifische Wärme- oder Kälteempfindung durch mechanische oder elektrische Reizung der Temperaturflecken auszulösen.

In bezug auf Lokalisierung war es interessant, dass Reizung eines Fleckens im Vorderarm eine etwas weit irradiierende Empfindung an dem Orte des gereizten Fleckens hervorrief und auch eine diffuse Empfindung, die sich auf einen entfernteren Ort bezog, nämlich einen Ort in der Region des Metakarpus des Daumens. Das Gegenteil geschah häufig bei Erregung der Basis und des Daumenrückens. Ähnlich erzeugte Reizung des zweiten und dritten Gelenkes mit einer kalten Röhre nicht nur lokal eine Kälteempindung, sondern auch an dem Kopfe des ersten Metakarpus und vice versa. Es 
gab Variationen mit den Bedingungen, aber manchmal war die lokale Empfindung die stärkere, manchmal die schwächere. Diese Areale, auf welche die Empfindung bezogen wurde, waren niemals zufällig und gingen nie von protopathischen Arealen zu gesunden, noch von diesen zu protopathischen Arealen. Die einmal erweckte mangelhafte Lokalisation wird, wenn uur protopathische Sensibilität in Tätigkeit gesetzt worden ist, häufig mehr oder weniger im Bewusstsein durch das gelegentlich ins Spiel bringen der tiefen Sensibilität aufgewogen (durch Druck auf tiefe Strukturen bei der Applikation des reizenden Gegenstandes).

In diesen Fällen kann anscheinend protopathische Sensibilität, dank der begleitenden Wirkung des anderen peripheren Nervenmechanismus, gut lokalisiert werden; es scheint jedoch einen Kampf zwischen der durch tiefe Sensibilität erweckten Lokalisation, die, wenn sie allein wirkt, genau ist und den diffusen und irradiierenden Eigenschaften der protopathischen Lokalisation zu. geben.

\section{Anmerkung:}

Um die von der tiefen Sensibilität herrührende Lokalisation zu vermeiden, benutzten Head und Rivers (40) in manchen Fällen winzige Tropfen von Äther oder von Äthylchlorid anstatt einer eiskalten Röhre. Die charakteristischen in Irradiierung und Lokalisation am entfernten Orte bestehenden Eigenschaften der protopathischen Empfindungen der Kälte erschienen dann ungestört von den Folgen des Druckes. Ein solcher dem Handgelenk applizierter Reiz kann in dem ganzen applizierten Areal auf dem Handrücken, den grösseren Teil des Daumens mit inbegriffen, das Gefühl der Eiseskälte verursachen; und auf Reizung einer Gruppe von Punkten am Vorderarm folgte eine intensive Kälte über die ganze dorsale Oberfläche des Daumens.

Mit der Rückkehr der Empfindung für leichte Berührung usw. in einem vorher nur mit protopathischer Sensibilität begabten Areal nehmen die stechenden irradiierenden Eigenschaften der erzeugten Empfindung und die mangelhafte Lokalisation allmählich, obgleich ziemlich rasch ab und verschwinden.

Daher können die wesentlichen Eigenschaften der protopathischen Sensibilität folgendermassen zusammengefasst werden: Der nur mit protopathischer Sensibilität begabte Teil reagiert nur auf schmerzhafte Impulse, sowie sie durch Nadelstiche, Ziehen oder Verschieben der Haare, die Extreme der Hitze (über $38^{\circ} \mathrm{C}$ ) und der Kälte (unter $24^{\circ} \mathrm{C}$ ) erzeugt werden und gibt eine diffuse, mangelhafte Lokalisation. Ferner irradiiert jede Art der erweckten Empfindung weit und hat eine stechende Eigenschaft. Überdies scheint es auch in diesen Arealen während der früheren Stadien, die sich über viele Monate oder ein oder zwei Jahre ausdehnen, dass der notwendige 
Reiz, um Empfindung hervorzurufen, grösser sein muss (d. h. es existiert eine höbere Schwelle) und die entsprechende Empfindung ist (neben dem irradiierenden und stechenden Charakter) intensiver und lebhafter als an den normalen Teilen, sie erwacht langsamer (latente Periode) und dauert länger (Nachwirkung) als über den normalen Teilen.

\section{Epikritische Sensibilität.}

Es ist bereits festgestellt worden, dass in einem Areal eines durchschnittenen afferenten Nerven, der genäht worden ist, protopathische Sensibilität oder Reaktion auf bestimmte Reizarten (nämlich auf Nadelstiche, extreme Hitze und Kälte) 7-10 Wochen nach Durchschneidung und Naht wiederzukommen beginnen, und dass das Vermögen, diese Reizarten vollkommen und leicht zu erkennen, durchschnittlich in ungefähr 26 Wochen vollständig ist. Ungefähr 50 Wochen nach der Verletzung fängt jedoch eine andere Reibe von feineren und zarteren Gefühlen an allmählich in demselben Areal zurückzukommen und ist durchschnittlich in ungefähr ein bis zwei Jahren vollständig; es sind dies das Erkennen der leichtesten Berührungen mit einem Baumwollwischer, das gleichzeitige Erkennen der Zirkelspitzen, kleine gewöhnlich warm genannte Temperaturunterschiede (d. h. zwischen $26^{\circ}$ und $37^{\circ} \mathrm{C}$ ) und auch das Vermögen der genauen Lokalisation. Diese Gefüble sind in ihrem Wiederkommen in bezug auf Intensität und Zeit so nahe assoziiert, dass Head (3) sie unter dem Ausdruck epikritische Sensibilität gruppiert hat. Wenn diese Gefühlsarten besser werden, verschwinden die besonderen Eigenschaften der oben beschriebenen protopathischen Sensibilitäten, wie Stechen, Ausstrahlung und Intensität, so dass z. B. ein Nadelstich Schmerz hervorruft, Eis die Kälteempfindung, wie bei einer normalen, gesunden Haut.

Das Verschwinden der Tendenz, die Empindung in entfernte Teile (siehe Seite 672) zu verlegen, war das auffallendste Zeichen für das Wiederkommen der Sensibilität auf Tasthautreize. Baumwolle über die Haare gebürstet, verursachte kein sonderbares diffuses Stechen mehr und wurde von der Haut selbst erkannt, wenn der Vorderarm sorgfältig rasiert war. Überdies reagierten im wiederhergestellten Areal der Sensibilität eine Reihe von Punkten nicht nur auf v. Freys Tasthaare Nr. 5, sondern selbst auf Nr. 3. Das Wiederkommen dieser Sensibilität wurde, wie schon betont, im Winter verzögert und machte wäbrend des Sommers gute Fortschritte. Die ersten Zeichen der Empfindlichkeit für Baumwolle traten in Heads Vorderarm 365 Tage nach der Operation auf und verbreiteten sich bis zu seinem Handrücken 568 Tage nach der Operation. Genaue Lokalisation von leichten Berührungen wurden dann möglich. Ferner wurde die spitze Natur irgend einer scharfen Spitze (Nadel) als solche erkannt (Acuaesthesia) ohne oder mit 
irgend, einer anderen spezifischen Empfindung, welche sonst durch sie entsteht. Daher war, ganz abgesehen von der Druck-Berührung oder dem Schmerz, die Unterscheidung zwischen der Spitze und dem Kopf der Nadel sofort möglich.

\section{Temperatur-Sensibilität.}

Temperaturen, gegen welche protopathische Teile unempfindlich waren, erweckten nun eine Empfindung und sowohl Ausstrahlung wie Lokalisierung der Temperaturempfindung hörte auf. Teile, welchen eine Temperatur von $36^{\circ} \mathrm{C}$ oder selbst $34^{\circ}$ und niedriger appliziert wurden, gaben eine Wärmeempfindung. Diese unterschied sich wesentlich von der von den Wärmeflecken erhaltenen Hitzeempfindung. Denn sie war gut lokalisiert und wurde von Teilen ausgelöst, wo keine Wärmeflecken entdeckt werden konnten, jedoch reagierten die Wärmeflecken, obgleich sie noch auf Temperaturen von $38^{\circ} \mathrm{C}$ und mehr reagierten, auf niedrigere Temperaturen nicht. Eine analoge, jedoch weniger auffallende Veränderung fand bei sogenannten kühlen Temperaturen statt. Es war daher klar, dass regenerierende Teile des Vorderarms und der Hand empfindlich gegen Temperaturen wurden, die keine Reaktion in jenen Teilen hervorriefen, die nur mit Wärme- und Kälteflecken begabt sind. Diese erböhte Empfindlichkeit gegen Temperaturreize war nicht von der Vermehrung der Anzahl an Wärme- und Kälteflecken begleitet, sondern von einer verminderten Lebhaftigkeit der Reaktion auf alle Arten der T'emperaturreizung eben so wie von einer Hemmung der Lokalisierung in entfernte Teile. Ferner war es in den frühesten Stadien der epikritischen Regeneration möglich, indem man den affizierten Teil auf Eis legte, zeitweise die irradiierenden und lebhaften Eigenschaften der protopathischen Sensibilität zurückzubringen, während das Wärmen der Hand eine Rückkehr der epikritischen mit Verschwinden der reinen protopathischen Phänomene erzeugte.

\section{Anpassung an Hitze und Kälte:}

Es ist schon festgestellt worden, dass in einem protopathischen Areal, in welchem Regeneration der epikritischen Sensibilität vorkommt, eine der frühesten bemerkbaren Veränderungen, die Verminderung oder Vernichtung der irradiierenden und entfernt lokalisierenden Eigenschaften der im protopathischen Areal erbältlichen Empfindungen ist. Applikation der Kälte wird nun, wie z. B. durch Legen des Teiles auf Eis für ein paar Minuten, wie es im Winter manchmal vorkommt, die Empfindlichkeit der Haut reduzieren, so dass der Teil reagiert wie in dem früheren Stadium der Erholung, d. h. mit den irradiierenden und stechenden Eigenschaften des protopathischen Typus. Es wurde von Head und Rivers (40) gefunden, dass, wenn man den zu untersuchenden Teil für ein paar Minuten in kaltes Wasser oder in 
heisses Wasser legt, die Oberflächentemperatur der Haut nach Wunsch innerhalb bestimmter Grenzen $\left(20^{\circ}\right.$ bis $\left.31^{\circ}\right)$ erhöht oder erniedrigt werden kann. Dazu wurde gefunden, dass die Temperatur des Reizes, auf welche ein Teil eine "neutrale" Reaktion gab (d. b. weder heiss noch kalt) auch erhöht oder erniedrigt werden konnte. Mit anderen Worten passte sich der Teil der Wärme oder der Kälte an. Dies kam jedoch nicht in einem protopathischen Areal vor, dessen neutraler Punkt der thermalen Sensibilität tatsächlich selbst in einem der Wärme oder Kälte adaptierten Teile konstant blieb.

Wenn so die normale Hand der Wärme adaptiert ist, wird $33^{\circ} \mathrm{C}$ der neutrale Punkt, $35^{\circ} \mathrm{C}$ scheint warm und $31^{\circ} \mathrm{C}$ kalt zu sein. Sorgfältig der Kälte adaptiert, verschiebt sich der neutrale Punkt auf $27^{\circ} \mathrm{C}$ und alle Temperaturen über $28^{\circ} \mathrm{C}$ werden als warm bezeichnet.

Die höchste Temperatur, auf welche Kältepunkte reagieren, ist $26^{\circ} \mathrm{C}$ und selten über $24^{\circ} \mathrm{C}$, und die niedrigste, auf die Hitzepunkte (oder der rein protopathische Teil) reagieren, ist $37^{\circ} \mathrm{C}$ und selten unter $40^{\circ} \mathrm{C}$. Es ist daher offenbar, dass irgend ein Mechanismus in der normalen Haut existieren muss, durch welchen eine Kälteempfindung bei Temperaturen zwischen $26^{\circ} \mathrm{C}$ und $37^{\circ} \mathrm{C}$ hervorgerufen wird. Ferner beseitigen diese $\mathrm{Be}$ obachtungen die von Head und Sherren (42) erfahrene Schwierigkeit, in. dem sie das Vorhandensein der Empfindung der Kühle, abgesehen von der Reaktion der Kälteflecke, beweisen.

Von besonderem Interesse in diesem Zusammenhang sind die Ansichten von Schwimmer, Cartier, Brissaud, Rendu usw., die glauben, dass sensorische Störungen eine fleckenförmige Anordnung haben und diejenigen von Boucour, Bourneville, Champion, Féré, Gerhardt, Grasset, $V$ edel usw., die, soviel man aus ihren Arbeiten ersehen kann, glauben, dass eine diffuse Anordnung vorkommt. Man sieht oben, dass Head und Rivers das Vorhandensein sowobl von fleckenförmigen, wie von diffusen Systemen bewiesen haben.

\section{Zirkelprobe:}

Nur in Gegenwart der tiefen Sensibilität zeigte der Pạtient kein Vermögen, die gleichzeitige Applikation der beiden Spitzen eines Zirkels zu erkennen, noch eine Tendenz eine Spitze als zwei zu bezeichnen. Mit dem Auftreten der protopathischen Sensibilität wurden die Angaben bei Zirkelversuchen unregelmässig; zwei gleichzeitige Punkte wurden oft als einer und ein Punkt als zwei bezeichnet. Dies hing in diesem Zustand offenbar von der Tendenz zu irradiieren und zum diffus werden $a b$, und während unter dieser Bedingung zwei $9 \mathrm{~cm}$ entfernte gleichzeitig applizierte Spitzen häufig als eine bezeichnet wurden, wurden, wenn epikritische Sensibilität dazu kam, keine Fehler gemacht, bis die normale Schwelle fast erreicht war, indem man die beiden 
Spitzen auf $2 \mathrm{~cm}$ einander näherte. Eine auffallende Wirkung bei der Wiederkehr der epikritischen Sensibilität ist daher die Reduktion der Entfernung zwischen zwei Zirkelspitzen, bei welcher eine oder zwei Zirkelspitzen eine äbnliche Empfindung erzeugen.

\section{Die Überlagerung. Vergleich der beiden.}

Aus der Untersuchung von einer Reihe von Fällen waren Head und Sherren (4) imstande, zu zeigen, dass durch Durchtrennung des Nervus medianus oder des Nervus ulnaris das gegen leichte Berührung unempfindlich gemachte Areal wenig an Umfang varijerte, während in scharfem Kontrast hierzu das gegen Stiche unempfindliche Areal beträchtlich varïerte. Ferner varieren die Areale der Hautanalgesie und der Tastanästhesie unabhängig voneinander. Dieser Mangel an Beziehung zwischen dem Umfang des Verlustes der Stichempfindung und der gegen leichte Berührung nach vollkommener Durchtrennung eines Nervens macht es unwahrseheinlich, dass die beiden Arten der Sensibilität von demselben ana tomischen System von Nervenfasern und Endorganen herstammen.

Ferner kommt nach einer gelungenen Naht eines durchtrennten Nerven die protopathische Sensibilität nicht nur zuerst wieder, sondern die ganzen affizierten Teile können mehrere Monate lang ganz unempfindlich gegen alle höheren Reize bleiben (leichte Berührung, feine Temperaturabstufungen, Zirkelprobe und sie sind der feinen Lokalisation unfähig). Und obgleich bei einem nur gequetschten Nerven (nicht einem durchtrennten) die Besserung eine allgemeine ist und die taktile Anästhesie und Analgesie zusammen Schritt für Schritt verschwinden, ist es schwierig, die Tatsachen der Dissoziation der Sensibilitäten und der oben erwähnten Erholungszeit zu verstehen, ausser unter der Anuabme, dass die protopathische und epikritische Sensibilität von zwei unabhängigen Reihen von Nervenfasern versorgt werden. Andere Tatsachen unterstätzen auch diese Ansicht. Protopathische Sensibilität kann mehr oder weniger unter Bedingungen, wie z. B. bei dem Vorhandensein von Fasergewebe zwischen den genähten Nervenenden, wieder hergestellt werden, die die Erneuerung der epikritischen Sensibilität abschwächt und verhindert. Auch die zur protopathischen Regeneration erforderliche Zeitdauer ist unabhängig von der Entfernung der Wunde von der Peripherie, während die zur epikritischen Rückkehr erforderliche Zeit von der Länge des zu regenerierenden Nerven abhäugt (5). Wenn wiederum zwei Systeme von Nervenfasern diesen beiden (protopatischen und epikritischen) Sensibilitätsarten dienten, sollte man Beispiele für die Veränderung der Verteilung dieser Systeme erwarten, so dass die umgekehrte Form der dissoziierten Sensibilität entstehen würde; mit anderen Worten, dass ein gegen Stiche unempfindlicher Teil auf die leichteste Berührung reagieren würde. Eine solche Dissoziation, das Vorhanden- 
sein der epikritischen Sensibilität und das Fehlen der protopathischen Sensibilität, obgleich relativ selten, wurde von Head und Sherren in mehreren Beispielen beim Menschen gefunden, und sie scheint besonders nach Durchtrennung der hinteren Wurzeln, welche den Arm versorgen, zu existieren. In einem solchen Falle war ein Areal über dem Deltoideus unempfindlich gegen Stiche und Eis, aber empfindlich, selbst nach dem Rasieren, gegen Baumwolle und die geringeren Grade von Hitze und Kälte. In einem anderen Falle war ein kleines Areal auf dem Handrücken empfindlich für Baumwolle und Wärme, aber unempfindlich gegen Stiche und Eis.

Überlagerung: Es ist schon gezeigt worden, dass die epikritische Verlagerung der Nervi medianus und ulnaris in der Handfläche ein wenig übereiuandergreift. Die Grenze der epikritischen Versorgung für den Medianus und Ulnaris (siehe Diagramm) geht durch den Ringfinger. In keinem Beispiel versorgte jedoch nach Durchtrennung einer dieser Nerven der bleibende Nerv den ganzen Ringfinger mit epikritischer Sensibilität oder mit anderen Worten, diese beiden Nerven können, so weit ihre epikritische Versorgung in Betracht kommt, einander nicht innerhalb der Breite eines einzigen Fingers überlagern. Ähnlicherweise stimmen bei den Handballen die Grenze der epikritischen Versorgung des Medianus und die des kombinierten radialen und äusseren Hautnerven tatsächlich überein, während auf dem Rücken des Zeigefingers und des Mittelfingers die epikritische Versorgung des Medianus die der präaxialen Gruppe (radiale und äussere Haut) um weniger als Dreiviertel des Umfanges der basalen Phalanx überragt. Im Vorderarm trennt eine scharfe Grenze die epikritische Versorgung der preaxialen von der der postaxialen Gruppe, sowohl an der Beuge- wie an den Streckoberflächen des Vorderarms. Die einzelnen Zweige, aus welchen die präaxiale Gruppe zusammengesetzt ist, greifen jedoch beträchtlich übereinander und Zerstörung von einem von ihnen führt zu keinem gut abgegrenzten Areal epikritischer Anästhesie. So scheint der Bestand des epikritischen Systems in den peripheren Nerven des Vorderarms und der Hand in Einheiten zerlegbar zu sein, welche entsprechen: 1. dem Ulnaris und inneren Hautnerven, 2. dem Medianus, 3. dem Rest der preaxialen Gruppe (radiale und äussere Hautnerven). An diesen erzeugen Läsionen Areale epikritischer Anästhesie, deren Grenze tatsächlich mit der der bleibenden Sensibilität übereinstimmt, die von dem angrenzenden Nerv herrührt, und deshalb kommt nur geringe oder keine Überlagerung der epikritischen Sensibilität dieser peripheren Nervengruppen vor. Aber je näher die Läsion an den hinteren Wurzeln liegt, um so weniger bestimmt sind die gegen epilkritische Reize unempfindlichen Grenzen des Areals und um so grösser ist das Übereinandergreifen der verletzten Stränge oder Wurzeln.

Die Anordnung der Verteilung der protopathischen Sensibilität ist jedoch von Grund aus verschieden. Durchtrennung der N. medianus 
und ulnaris erzeugt Verluet der protopathischen Sensibilität über ein verhältnismässig kleines Areal mit unbestimmten Rändern, und das Sensibilitätsareal für protopathische Reize nach Durchtrennung des N. medianus erstreckt sich in vielen Fällen über die ganze Handfläche. Wenn überdies, wie in einem Falle von Head und Sherren (7) der N. ulnaris erregt wurde, verbreitete sich die Empfindlichkeit über die Handfläche nach dem Daumen und der Basis des Zeige- und Mittelfingers. Daber zeigt sich als Folge der Durchtrennung oder der Erregung der protopathischen Nerven, dass sie in einem relativ ungeheuren Umfang übereinandergreifen. Ein Blick auf Figur 5 wird die Belege davon geben. Man sicht, dass der Ulnaris und der innere Hautnery sich bis zu einer Linie ausdehnen, die ungefähr der Sehne des Mittelfingers entspricht. Bei Durchschneidung dieser Nerven wird nur die protopathische Sensibilität über einen veränderlichen am inneren Rand der Handfläche und des kleinen Fingers (nicht arn Ringfinger) verloren; jedoch Durchschneidung nur des Medianus zeigt, dass die bleibende protopathische Sensibilität, die von dem undurchschnittenen N. ulnaris stammt, sich wohl über die Handfläche und über den ganzen Ringfinger, mit Ausnahme von einem Drittel der radialen Seite der beiden Endphalangen, ausbreitet. Wiederum erzeugt Durchschneidung am Vorderarm, Durchtrennung der radialen und äusseren Hautnerven, ein Areal protopathischer Unempfindlichkeit, das annähernd, ausser an dem Handgelenk, dem epikritischen Verlust entspricht; aber an dem Handgelenk greifen die protopathischen Fasern der post- und präaxialen Gruppen stark übereinander und der Verlust der Empfindung auf Stiche ist weniger ausgedehnt als der auf leichte Berührung.

Als ein Resultat dieses deutlichen Übereinandergreifens der Nervenfasern, welche der protopathischen Sensibilität dienen, verursacht Durchtrennung des peripheren Nervens Verlust der protopathischen Sensibilität über ein kleineres Areal, als das der begleitenden epikritischen Sensibilität. Wenn aber der Nerv zentral zu dem Bronchialplexus oder in den hinteren Wurzeln selbst durchschnitten wird, dann verhalten sich diese unempfindlichen Areale umgekehrt an Ausdehnung, d. h. das Areal der protopathischen Unempfindlichkeit überragt an Umfang das der epikritischen. Mit anderen Worten, je näher die trennende Läsion an den hinteren Wurzeln ist, um so ausgedehnter und bestimmter ist der Verlust der protopathischen Sensibilität, während je näher die Läsion eine der oben beschriebenen Nervengruppen, wie die mediane, ulnare oder die präaxiale Gruppe, trennt, um so bestimmter und umfangreicher ist der epikritische Verlust. Jedoch selbst die protopathischen Fasern von zwei aneinandergrenzenden Wurzeln greifen in ihren respektiven Hautfeldern übereinander, aber die Überlagerung dieser Fasern ist beträchtlich geringer als für diejenigen, welche die epikritische Sensibilität versorgen. 
Wenn wiederum es vorkommt, dass das mit einem peripheren Nerven versorgte Areal mit dem von einer oder mehreren hinteren Wurzeln übereinstimmt, dann würde Durchtrennung dieses Nerven einen Flecken totaler Hautsensibilität erzeugen, in welchem die Areale der protopathischen und epikritischen Unempfindlichkeit sich decken. Wie Head und Sherren zeigen, ist dies gut im Fall des Popliteus externus, einschliesslich seines lateralen Hautzweiges zu sehen. Durchsehneidung dieses Nerven erzeugt ein Areal von epikritischem Verlust, das durch eine Linie begrenzt ist, welche schräg über das Schienbein (vergl. auch Sherrington, loc. eit.) bis zu einem Punkte gerade vor dem inneren Malleolus verläuft und in der Region der Wade vertikal hinunter entlang der Achillessehne. Dasselbe Areal umgrenzt den Verlust für protopathische Sensibilität, so dass eine bemerkenswerte Übereinstimmung dieser beiden Areale ensteht.

\section{Anmerkung:}

Von Interesse in diesem Zusammenhang ist das anästhetische Areal, welches von Camus und Sézary (loc. cit. S. 538) als ein Resultat der Wurzelaffektion der fünften Lumbal- und der ersten Sakralwurzel angegeben wird. Wie sie es darstellen, ist das affizierte Areal unter dem Knio scheinbar identisch mit dem, welches Head und Sherren als das Ergebnis der Verletzung des äusseren Nervus popliteus aufgezeichnet haben. Die selben Forscher behaupten (S. 297), dass die wechselseitige Beziehung der anderen Nervenzweige im Bein verwickelt ist, und kein klares Beispiel für die Gesetze, die sie niedergelegt haben, liefert. Ferner behauptet Mirallié (S. 14), dass in den unteren Gliedmassen die sensorische Wurzeltopographie so vollständig ist, dass sie jeder Beschreibung trotzt. Er behauptet auch, dass in affizierten Arealen die Ränder unvollständige Unempfindlichkeit und topographische Variationen an Intensität zeigen, z. B. sind die anästhetischen Phänomene nicht über das ganze Wurzelareal identisch. (Auch Camus und Sézary und Jeanselme).

Die anatomische Grundlage, welche auf dieses funktionelle Übergreifen Bezug hat, wird gut in anatomischen Werken gezeigt, wie in denjenigen von Krause, Brooks, Hédin, Frohse, Thane usw. und braucht hier nicht weiter betrachtet zu werden.

Aber in diesem Zusammenhang wollen wir uns einen Augenblick den Arbeiten von anderen über die sensorische Innervation der Haut zuwenden. Mit Hilfe von zwei verschiedenen Methoden, 1. durch Untersuchung des nach Durchtrennung einer oder mehrerer Rückenmarkswurzeln erbaltenen Anästhesieareals, 2. durch Untersuchung des Areals der „bleibenden Sensibilität", wenn eine Rückenmarkswurzel unversehrt gelassen worden ist, und ihre Nachbarn oben und unten durchtrennt sind, hat Sherington in einer Reihe von meisterhaften Untersuchungen (9. loc. cit. S. 758) gezeigt, dass, obgleich in 
einem Plexus jede hintere Rückenmarkswurzel mehrere Beiträge an mebrere Nervenstämme abgibt, die Hautverteilung der Wurzel nicht aus unverbundenen Flecken zusammengesetzt ist, sondern aus einem fortgesetzten Feld, dem sensorischen spinalen Hautfeld. Ferner erstreckt sich jedes von diesen bis zu einem gewissen Umfang über die angrenzenden Hautfelder. Jedes hat eine vordere Überlagerung, welche sich in die vor ihm liegenden (frontalen) segmentierten Felder erstreckt, jedes eine hintere Überlagerung in hintere Felder (kaudal). Die Vor- und Nachüberlagerungen sind sehr gross, und es scheint, als ob jeder Hautpunkt im ganzen Körper wenigstens von zwei sensorischen Rückenmarkswurzeln, in bestimmten Regionen von drei, versorgt wäre, und am äusseren $\mathrm{Ohr}$ möglicherweise sogar von vier. Die Zahl der Segmente, welche in die Komposition der Haut der Glieder eintreten, ist grösser, als die Zahl der Segmente, die zu deren Muskulauar beiträgt.

Als Beispiel einer dreifachen Wurzelversorgung zeigte Sherrington die Fusssohle der Katze, die Brustwarze des Affen und ein dreieckiges Hautareal auf dem Rücken und im Innern der Hand, welches von dem Cerv. 7 Cerv. 8 und dem ersten Thorakalnerven versorgt ist. Aber entlang dem Rücken des Stammes, ungefäbr auf halbem Wege zwischen den mittleren dorsalen und den mittleren lateralen Linien des Körpers, ist die Menge der Rückenmarkswurzelüberlagerung der folgenden Hautfelder geringer als auf der Hand. Beim Vergleich der Areale der sensorischen Innervation der peripheren Nervenstämme mit denjenigen der hinteren Wurzeln, stellt er übrigens fest (loc. cit. S. 109), dass sowohl in der Hand, wie im Fuss des Makakus der Umfang der Überlagerung der Hautfelder der peripheren Nervenstämme selbst auf den ausserordentlich empfindlichen Plantar- und Palmartlächen viel geringer ist, als der der Hautareale der Rückenmarkwurzeln; er ist tatsächlich nicht so gross wie die Überlagerung der Felder der Nervenwurzeln sein kann, die drei Segmente voneinander entfernt sind.

Nach Head (loc. cit. S. 294) scheint die Versorgung des epikritischen Systems in folgenden Einheiten in den peripheren Nerven zu bestehen: 1. Ulnaris und Cutaneus internus, 2. medianus und 3. radialis und Cutaneus externus, während die protopathische Einheit in ein oder mehreren hinteren Wurzeln gesucht werden muss und die vollständige Übereinstimmung zwischen den Ergebnissen von Heads Untersuchungen am Menschen und Sherringtons am Affen sind daher auffallend.

Bei einer Untersuchung über die spinalen Wurzeln und die dissoziative Anästhesie beim Affen kommt Sherrington zu einigen auffallenden Schlüssen. Er findet, dass die Zerstörung der Hautempfindung bei Makakus durch Durchtrennung der spinalen Wurzeln, nicht nur in gewissem Umfang eine dissoziative ist, sondern dass wenigstens bei bestimmten Rückenmarksnerven Durchschneidung jeder spinalen Wurzel in einem begrenzten Haut- 
areal, das viel kleiner als das ganze Hautfeld der Wurzel ist, eine Vernichtung der Wärmeempfindlichkeit ohne Vernichtung, aber mit einer begleitenden Abschwächung der Tastempindlichkeit hervorruft. Er findet ferner, dass das Schmerzfeld und das Wärmefeld einer einzigen sensorischen Rückenmarkswurzel wenigstens bei bestimmten spinalen Wurzeln jedes einzelne weniger ausgedehnt ist, als es das Berührungsfeld derselben Wurzel ist und dass die Wärmefelder und Schmerzfelder dieser Wurzeln in manchen Hautregionen mehr übereinandergreifen, als in anderen, z. B. in der Hand und im Fuss mehr als in Teilen des Vorderarmes, des Schenkels und des Rumpfes. Er stellt auch fest, dass in einem Hautfeld, wo nur eine Wurzel bleibt, punktförmige Reizung am Rande weniger mit Schmerz verbunden ist, als die näher am Zentrum des Feldes.

In einer wertvollen Mitteilung über uniradikuläre Lähmung des brachialen Plexus bestätigt Buzzard beim Menschen die hauptsächlichsten strittigen Punkte von Sherrington beim Affen. Buzzard (S. 301 u. 315) beschreibt mehrere Krankheitsfälle, die auf eine Rückenmarkswurzel beschränkt waren, aus weichen nicht nur eine dissoziative Anästhesie, sondern gegen Schmerz, Hitze und Kälte unempfindliche Areale resultieren, die ausgedehnter und ausgesprochener sind, als die entsprechende Abnahme gegen Tastsensibilität. Ferner betont er, dass jedes unempfindliche Areal geringer an Umfang ist, als das gesamte Hautgebiet der entsprechenden spinalen Wurzel, und S. 301 zeigt er, dass in wenigstens einem Falle die Spitze einer Nadel nicht als scharf erkannt wurde, mit anderen Worten, dass Anästhesie, das tactile Erkennen der Spitze einer Nadel als Gegensatz zu ihrem Kopf fehlte ${ }^{1}$ ).

Aus den obigen Versuchen beim Affen und der Krankheit beim Menschen folgt, dass die Nerven in einer Rückenmarkswurzel, die protopathischer Sensibilität dienen, eine geringere Überlagerung in ihrem Hautareal zeigen, als die entsprechenden epikritischen Nerven (während das Umgekehrte bei gewissen peripheren Nerveneinheiten vorkommt). Dieses Ergebnis steht in auffallender Übereinstimmung mit der späteren und detaillierten Mitteilung, die oben von $\mathrm{Head}$ und Sherren gegeben wurde.

Die Arbeit von Petren und Bergmark (75) bestätigt von einem anderen Standpunkt aus in der Hauptsache die obigen Resultate. Sie zeigen in einer Reihe von zwölf oder mehr Fällen beim Menschen, dass nicht nur verschiedene Grade der Hyperalgesie und Hypoalgesie in demselben Fall von idiopathischem Herpes zoster existieren können, bei welchen, wie ursprünglich von Head und Campbell (39) gezeigt wurde, der Sitz der Läsion im spinalen Ganglion ist, sondern dass gegen Schmerz, Hitze und Kälte unemp-

1) Raymond hat gezeigt, dass eine Haematomyelia, Dejérine, dass eine Läsion des Hinterhorns, und Loehr, dass eine Syringo-myelia eine Wurzelverteilung der affizierten Areale geben kann. Aber in solehen Fillen ist es wichtig, festzustellen, ob die Symptome von einer primären oder sekundären Läsion herrühren. 
findliche Areale und Flecken in Fällen ohne besondere Affektion auf Berührung vorkommen. Durch verschiedene Beobachtungen sind sie imstande, $\mathrm{zu}$ behaupten, dass Innervationsareale der verschiedenen thorakalen Spinalganglien bezw. spinalen Wurzeln, die sich auf Schmerz und Temperatur beziehen, viel weniger überragen als die der Berührung. Ferner zitieren sie Féré (26), Grasset und Vedel (37), Brissaud (13) und Delille und Camus, um zu zeigen, dass wenn Berührung in einem solchen Falle von Analgesie bei Herpes zoster sicher abgeschwächt wird, die Affektion die Spinalganglien von wenigstens drei oder mehr Segmenten in sich schliessen muss.

Andere Forscher, besonders Furier und Yeo, Forgue und Lannegrace, Risieu Russell, der Verfasser und viele andere haben auch experimentell Gebiete abgegrenzt, die von den sensorischen (und motorischen) Wurzeln innerviert werden, da aber ihre Resultate mit den oben angegebenen Tatsachen übereinstimmen, braucht hier nicht darauf eingegangen zu werden. Klinisch haben eine Menge von Forschern Duchenne, Erb, Charcot, Déjérines, Hitzig, Laehr, Chipault, Gowers, LortatJacob, Langevin, Ramon usw. unsere Kenntnis des Gegenstandes gefördert. Bemerkenswert unter vielen wichtigen Arbeiten ist die von Head und Campbell (39), welche die trophischen Feldern der verschiedenen Spinalganglien abgrenzten, Thorburn, Kocher usw.

Von grossem Interesse in Verbindung mit obigen Ähnlichkeiten und Verschiedenheiten zwischen afferenten Spinalwurzeln und afferenten peripheren Nervensensibilitäten sind die Resultate von verschiedenen Forschern über die relative Bedeutung der proximalen und distalen Repräsentation in bezug auf die tiefen und Hautsensibilitäten. Es ist ferner von zahlreichen Autoritäten, namentlich von Frenkel, Förster, Lewandowsky usw. gezeigt worden, dass das Fehlen oder die Verminderung der afferenten Regulation eine beträchtliche Abnahme im Muskeltonus und in der Koordination erzeugt. Dies ist z. B. in Fällen von Tabes usw. gut bekannt. Ferner führt Unterbrechung des Tractus dorso-spino-cerebellaris (von Flechsig) oder des Tractus ventrospino-cerebellaris (von Gowers), wie von Bing, Marburg, Page May usw. gezeigt wurde, zu einer deutlichen Hypotonizität der mit ihnen in Beziehung stehenden Muskeln, und selbst Läsionen hestimmter Teile des Grosshirns (vermis usw.) führen, wie von. Gordon Holmes, Stewart und anderen erwiesen wurde, zu einem ähnlichen Resultat. Aber eine der auffallendsten Tatsachen ist, dass eine Störung der Bahn der tiefen Sensibilität Hypotonizität erzeugt, die an den proximalen und grösseren Gelenken deutlicher, und weniger deutlich an den kleineren distalen Segmenten des Gliedes ist, während man wohl weiss, dass bei den Sensibilitäten des Hautsystems das Umgekehrte der Fall ist, da die Deutlichkeit des sensorischen Erkennens in den distalen Arealen des Gliedes viel grösser ist als in den proximalen. 
In diesem Zusammenhang ist ein neuerer Versuch von Sherrington von grossem Interesse. Dieser Forscher zeigte auf dem internationalen Physiologenkongress in Heidelberg 1907 eine Katze, bei welcher er alle Nerven, welche die Haut der vier Extremitäten versorgen, vollkommen durchschnitten hatte. Er zeigte, dass das Tier noch mit Bestimmtheit gehen, über eine Leiter laufen konnte, und im ganzen schien es, als ob sie wenig, wenn überhaupt irgend eine Schwierigkeit bei ihren gewöhnlichen Bewegungen hatte. Später zeigte derselbe Forscher (bei einem "meeting of the Physiological Society at Cambridge“, Mai 1908) dasselbe Tier nach Durchschneidung von einer gewissen Anzahl von hinteren Wurzeln. Es zeigte sich ein bemerkenswerter Unterschied zwischen den beiden Zuständen, denn das Tier zeigte nun die äusserste Ataxie und Hypotonie, die viel deutlicher in den proximalen als in den distalen Gelenken waren.

Als ein Beispiel für manche der häufigen Täuschungen in der Art und Weise, die Empfindung zu prüfen, konnte nichts lehrreicher sein als diese Demonstration. Die Haut der vier Extremitäten war vollständig gefühllos gemacht, denn jeder afferente Nerv zu den Hautgebilden war vorher durch. schnitten worden; beim Prüfen der affizierten Areale auf die gewöhnliche Weise durch Applikation einer Berührung oder einer Nadel oder von etwas Heissem oder Kaltem, gab die Katze jedoch ein unzweifelhaftes und sofortiges Zeichen des Erkennens des Reizes. Die Reaktion rührte notwendigerweise von gleichzeitiger Reizung der Nerven her, welche die tiefe Sensibilität versorgen, indem diese Erregung durch den begleitenden Druck, der mit der Applikation der Reize assoziiert ist und gelegentlicb auch durch die mehr oder weniger geringe Veränderung in der passiven Lage von einem oder mehreren Teilen des Tierkörpers induziert war. Wenn das afferente Hautsystem allein erregt wurde, war niemals eine Spur des Erkennens durch das Tier beobachtbar. Der Fall zeigte, wie ausserordentlich fein der Mechanismus der tiefen Sensibilität ist, der auf jene Impulse reagiert, welche Veränderung in der passiven Lage eines Teiles erzeugen. Was für einen Fallstrick dieser Mechanismus bei vielen der gewöhnlichen Methoden der klinischen oder anderer Untersuchung darstellt, wird nur zu gut bei einer Durchsicht der widersprechenden Resultate, die so reichlich in der medizinisehen Literatur vorhanden, erkannt. Es gibt daher augenscheinlich ein tiefes afferentes System, das höchst empfänglich für Reaktionen ist, die sekundär im tierischen Organismus durch Veränderungen in dessen Umgebung erzeugt werden.

In jedem vielzelligen Tiere gibt es zwei Verteilungen der rezeptorischen (afferenten) Organe, von denen jedes ein Feld ist, das in gewissen Beziehungen fundamentell von dem anderen verschieden ist. Auf das Oberflächenfeld wirken zahllose Veränderungen seiner Umgebung. Es hat seit zabllosen Jahren den vollen Strom der verschiedenen Agentien gefühlt, die immer von der Aussenwelt auf es einströmen. Dieses Feld, extero- 
rezeptiv wie Sherrington es nennt, im Gegensatz zu dem propriorezeptiven, ist reich an Zahl und Verschiedenheit der Rezeptoren, die die Anpassung darin entwickelt hat. Es kommt besonders leichte Berührung, Steigerung und Abnahme der Temperatur, mechanische Reizung, wie ein Stich usw., das Erkennen von zwei oder mehr Punkten, feine Lokalisation usw. in Betracht.

Andrerseits ist die Erregung der Rezeptoren des proprio-rezeptiven Feldes, im Gegensatz zu jenen des extero-rezeptiven Feldes, nur sekundär mit den Wirkungen der Umgebung verbunden. Die proprio-rezeptiven Rezeptoren erhalten ihre Reizung durch irgend eine Tätigkeit, z. B. eine Muskelkontraktion, die selbst eine primäre Reaktion auf Erregung eines Oberflächenrezeptors durch die Umgebung war. Die primäre Reaktion wird in der Mehrzahl der Fälle in einem Rezeptor des extero-rezeptiven Feldes erregt. Daher entstehen Reflexe (durch welche man allein auf die Resultate der Reizung bei niederen Tieren schliessen kann) sekundär durch proprio-rezeptive Organe und werden gewöhnlich mit gewissen durch extero-rezeptive Organe erregte Reflexe verknüpft. Mit anderen Worten erregt die Reaktion der Tiere auf Reizung eines ihrer Exterorezeptoren bestimmte Gewebe und die auf diese Weise erzeugte Tätigkeit in den letzteren erregt in ihnen ihre Rezeptoren, welche Propriorezeptoren sind. Auf diese Weise verändert eine Muskelbewegung, welche durch einen Reiz der Haut eines enthirnten Tieres erzeugt wurde, die Form und Spannung der Muskeln, Gelenke usw., welche die Rezeptoren in diesen Strukturen erregen und diese wiederum erzeugen einen Reflex in ihren eigenen Bogen und ihre Reaktion hat eine "alliierte" Beziehung zu der durch die Haut erregte Reflexreaktion. Es könnten zahllose Beispiele davon angeführt werden, aber es genügt für unseren gegenwärtigen Zweck festzustellen, dass, wie Sherington gezeigt hat, der Reflex. aus dem Muskelgelenkapparat in vielen Fällen sowie der Kratzreflex, den durch die Haut erzeugten Reflex zu verstärken erseheint, während er in anderen die von der Haut eingeleitete Tätigkeit lähmt oder hemmt. Z. B. ist Reflexhemmung der Beuger des Knies beim Rückenmarkshund regelmässig durch Reizung der Haut einer Zehe des gekreuzten Hinterfusses auslösbar. Dazu kommt zu der Kategorie der "alliierten exzitatorischen" Reflexe, eine Kategorie der „alliierten hemmenden" Reflexe. Vielleicht ist noch eine Illustration dieser alliierten Reflexe durch eine der jüngsten Entdeckungen hier am Platze. Sherrington zeigte bei der "Meeting of the British Association of Science in Dublin 1908", wie bei dem enthirnten Tiere, bei welchem in beiden Beinen der Quadriceps extensor-Muskel mit seinen efferenten und afferenten Nerven isoliert worden und nur vermittelst dieser Nerven in Verbindung mit dem Rückenmark war, wenn dann einer von diesen Muskeln passiv erschlafft wurde, sich der entgegengesetzte gekreuzte Muskel streckte, and wenn er gestreckt wurde, sich der entgegengesetzte kontrahierte. So er- 
zeugt in auffallender Weise passive Bewegung in einem Muskel abwechselnd eine alliierte erregende und hemmende Reflexbewegung, selbst in einem Muskel des entgegengesetzten Gliedes.

\section{Anmerkung 1:}

Man sieht auf S. 671, dass 152 Tage nach Durchschneidung eines Hautnerven die daraus entstandene Aualgesie und der Verlust der Kälteempfindung vollkommen über ein Areal verschwunden war, auf welchem vorher ein Geschwür nicht heilen wollte, aber mit der Wiederkehr der protopathischen. Sensibilität waren auch die Reaktion auf Schmerz und äusserste Kälte und Hitze, die trophischen Schwierigkeiten vergangen.

Ungefähr zu derselben relativen Zeit, wie sie bei anderen Fällen sich fand, fangen die Schweissdrüsen wieder an zu funktionieren. Diese trophischen und sekretorischen Störungen sind von vielen Forschern und besonders von Head, Rivers und Sherren mitgeteilt worden. Schlesinger (81) hat bestimmte Schweissbahnen im Verlaufe der sensorischen Nerven gezeigt, und obgleich, wie Sherring ton, Singer-Münzer, Münzer, Wiener, Kohnstamm usw. gezeigt haben, keine direkten zentrifugalen Fasern vom Säugetierrückenmark ausgehen und direkt durch die hinteren Wurzeln und Spinalganglien gehen, haben verschiedene Forscher die Existenz zentrifugaler Impulse bewiesen, welche direkt von den Spinalganglien nach der Peripherie gehen, z. B. Du-Bois Reymond, Exner, Gotch und Horsley, Stricker usw. und besonders die klassische Arbeit über vaso-dilatatorische Nervenfasern von Bayliss. Die Erzeugung der efferenten, motorischen und vasomotorischen Phänomene durch afferente Impulse, die von der Peripberie nach dem zentralen Nervensystem hinaufführen, ist schon erwähnt worden. Diese Fragen können nicht weiter in diesem Aufsatz erörtert werden.

\section{Anmerkung 2 :}

Eine ungeheure Forschungsarbeit ist der Empfindung der Eingeweide gewidmet worden, besonders von Mackenzie, Lennander, Müller, Head und vielen anderen. Die inneren Organe sind offenbar mit tiefer Sensibilität und protopatbischer Sensibilität niederer Art versehen. Diese können sich jedoch unter gewissen Bedingungen durch einen „Bahnungsvorgang" sehr aufdringlich gestalten. Aber der ganze Gegenstand ist so gross geworden, dass er eine besondere Bearbeitung erfordert und im vorliegendem Aufsatz nicht betrachtet werden wird.

So weit haben wir nun das Vorbandensein und die Anordnung der verschiedenen Mechanismen der Sensibilität in den peripheren Nerven betrachtet, wobei ihre Einflusssphären und ihre gegenseitigen Interreaktionen inbegriffen sind. Wir haben gesehen, dass, nach Head, in der Peripherie drei distinkte 
Systeme von Nervenfasern sind, welche der Leitung der afferenten Impulse dienen. 1. Jene der tiefen Sensibilität, welche die durch Druck erzeugten Impulse leiten und vermittelst deren übermässigen Druck, sogar Schmerz erregt werden kann; auch Impulse, welche dem Ortssinn und dem Sinne für aktive und passive Bewegung zugrunde liegen.

Die Fasern dieses Systems laufen hauptsächlich mit den motorischen Nerven und werden nicht durch Durchschneidung aller sensorischen Nerven wach der Haut zerstört, aber wenn sie durchschnitten sind, kann die Regeneration unter sechs bis sieben Monaten stattfinden.

2. Protopa this che Sensibiltät, welche die von schmerzlicher Reizung der Haut oder der Haare oder von extremer Hitze oder Kälte (unter $24^{\circ} \mathrm{C}$ und über $40^{\circ}$ G) stammenden Impulse übermittelt und eine diffuse Lokalisation erzeugt.

Verlust der protopathischen Sensibiltät vernichtet: Hautschmerz, besonders den durch Stechen, Brennen, Erfrieren usw. erzeugten.

Wärmeempfindungen durch Temperaturen um $40^{\circ} \mathrm{C}$.

Kälteempfindungen von Temperaturen unter $20^{\circ} \mathrm{C}$.

Diese Fasern regenerieren schnell nach Vereinigung der Enden der durchschnittenen Nerven, so dass die Empfindung innerhalb von 7 bis 10 Wochen wiederzukommen beginnt und in ungefähr 26 Wochen vollständig ist. Sie greifen auch bedeutend in ein Areal, das von angrenzenden Nerven versorgt ist, über. Mit der Rückkehr der protopathischen Sensibilität verschwinden auch verschiedene trophische und sekretorische Störungen.

3. Epikritische Sensibilität, welche das Vermögen des Erkennens von leichter Berührung, der Unterseheidung zweier Spitzen (Zirkelprobe) und der feineren, kühl und warm $\left(24^{\circ}\right.$ bis $\left.40^{\circ} \mathrm{O}\right)$ genannten Temperaturunterschiede vermittelt und feine Lokalisation gestattet.

Verlust der epikritischen Sensibilität vernichtet:

Wahrnehmung leichter Berührung über haarlosen oder rasierten Teilen. Hautlokalisation.

Unterscheidung von Zirkelspitzen.

Erkennen von Grössenunterschieden.

Unterscheidung von mittleren Temperaturgraden von um $25^{\circ}$ bis $40^{\circ} \mathrm{C}$.

Diese Fasern regenerieren langsamer als diejenigen, welche die protopathische Sensibilität versorgen, und nach Vereinigung kommt selbst unter den günstigsten Bedingungen die Empfindung nicht in weniger als 6 Monaten wieder. Die Verteilung dieser Fasern in den grösseren peripheren Nerven, wie in dem Medianius und Ulnaris, enthielt im Vergleich mit der grossen Überlagerung der protopathischen Versorgung eine sehr viel geringere Überlagerung. 
Es wäre nun von grösster Bedeutung, wenn auch kurz, die fundamentalen Anlagen zu betrachten, welche in den pächst höheren Neuronen für den Transport der afferenten Impulse vorkommen, nämlich im Rückenmark und Gehirn

„Regruppierung der afferenteń Impulse im Rückenmark".

Bei den peripheren Nerven sieht man, dass von den drei Systemen ein oder zwei Formen allein affiziert oder vernichtet werden können, und auf diese Weise kann man eine Dissoziation von bestimmten Impulsarten erhalten, die nur einer Form einer spezifischen Empfindung angehören.

\section{Schmerz:}

In einem Falle z. B. einer Flintenschusswunde des Plexus ischiadicus (11), war ein Areal an der äusseren Seite des Beines vorhanden, in welchem durch ein Nadelstich kein Schmerz ausgelöst werden konnte, aber er konnte ganz leicht vermittelst Druck ausgelöst werden, d. h. dass schmerzhafte Impulse durch das System der tiefen Sensibilität, aber nicht durch das protopathische vermittelt wurden, während aus einem Fall einer Läsion des Rückenmarks, die ein affiziertes Areal an der äusseren Seite des Beines im Gefolge hatte, ungefähr koextensiv wie im obigen Falle, gefunden wurde, dass Schmerz weder durch Nadelstich, noch durch Druck oder irgend eine Form der Reizung ausgelöst werden konnte.

Wenn durch eine Läsion des peripheren Nervensystems (hintere Wurzeln, Plexus oder Nerven) Schmerz, der von protopathischer Sensibilität herrührt, fehlt, dann fehlen die anderen Formen der protopathischen Sensibilität (äusserste Hitze und Kälte usw.) auch; wenn von übermässigem Druck stammender Schmerz fehlt, dann fehlen die anderen Formen der tiefen Sensibilität (Druck, Muskelsensibiltät usw.) auch, und der Schmerz kann nicht vollkommen fehlen, wenn nicht sowohl die protopathische wie die tiefe Sensibilität vernichtet sind, während $\mathrm{Head}$ und $\mathrm{Th}$ ompson imstande waren, in zahlreichen Fällen zu zeigen, dass wenn bei intramedullären Läsionen des Rückenmarks der Schmerz bei einer Reizart fehlt, er bei allen fehlt, d. h. dass alle Formen der schmerzhaften Reize gleichzeitig affiziert sind.

\section{Hitze und Kälte:}

Die Gruppierung der afferenten Impulse, welche zur Hitze- und Kälteempfindung führen, wenn sie von dem peripheren zu dem spinalen System übertragen wird, zeigt selbst noch deutlichere Kontraste. Wenn die Peripherie der epikritischen Sensibilität vernichtet ist, kann der Patient die mittleren Temperaturgrade $24^{\circ}$ bis $40^{\circ} \mathrm{C}$ nicht unterscheiden, aber er bleibt empfindlich gegen Temperaturen unter $20^{\circ} \mathrm{C}$ und über $45^{\circ} \mathrm{C}$. Nach Durchschneidung eines peripheren Nerven und der Rückkehr der protopathischen 
Sensibilität ist der Teil mit Sensibilität für Kälte unter $20^{\circ} \mathrm{C}$ und Hitze über ungefähr $40^{\circ} \mathrm{C}$ begabt; d. h. dass Zerstörung der epikritischen Sensibilität eine Reihe von (intermediären) Impulsen für Hitze und Kälte und Zerstörung der protopathischen Sensibilität in andere Reihe (für extreme Hitze und Kälte) vernichtet. Bei Hitze und Kälte werden, je nach dem affizierten peripheren System, andere besondere Impulse (Berührung, Schmerz usw.), wie oben auseinandergesetzt, affiziert.

Andererseits wird durch intermedulläre Läsion im Rückenmark, wenn Hitze für eine Reihe von Impulsen vermindert oder vernichtet wird, sie für alle vermindert oder vernichtet, und wenn Kälte für eine Gruppe von Impulsen vernichtet wird, ist es für alle. Die mittleren und extremen Temperaturen der Wärme und Kälte (im Rückenmark werden gewöhnlich, obgleich nicht notwendigerweise, Wärme und Kälte zusammen affiziert, müssen miteinander persistieren oder vernichtet werden, d. h. Unempfindlichkeit für Wärme und Kälte kann absolut sein, und doch kann der Patient imstande sein im selben Areal die leiseste Tastreizung wabrzunehmen und die beiden Spitzen des Zirkels zu unterscheiden. Solche Zustände können niemals von einer Läsion der peripheren Nerven allein herrühren.

\section{Leichte Berührung und tiefe Berübrung.}

Eine der häufig gesehenen charakteristischen Eigenschaften der peripheren Läsion ist die, dass leichte Berührungen nicht erkannt werden, während tiefe Berührung und Druck es werden. In solchen Fällen würden die letzteren Empfindungen, da sie von tiefer Sensibilität versorgt werden, notwendigerweise das Vorhandensein des Sinnes der passiven Lage und Bewegung in sich schliessen.

Wenn aber die Läsion intramedullär ist, gehen und kommen diese sensorischen Impulse zusammen. So (12) war in einem charakteristischen Fall von Brown-Séquard-Lähmung das ganze rechte Bein unempfindlich gegen Reizung mit Baumwolle, gegen von Freysche Haare und gegen Druck, selbst von vielen Kilogrammen. Dieser Patient erkannte jedoch jede passive oder aktive Veränderung in der Lage des sonst unempfindlichen Gliedes, d. h. die peripheren, afferenten Impulse für Druck und Berührung, welche durch sowohl epikritische wie tiefe Systeme gelangen, vereinigen sich im Rückenmark und müssen zusammen persistieren oder vernichtet werden. Im Rückenmark ist Tastempfindung wirklich ein Teil eines Druckes und Druck ist tatsächlich eine intensivere Form der Berührung; in der Peripherie können jedoch Berührung und Druck dissoziiert sein. Der beschriebene Zustand in dem oben erwähnten Falle des Fehlens dos Erkennens von leichter Berührung und Druck mit fortdauernder Wahrnehmung der passiven Lage kann nicht aus einer Läsion der peripheren Nerven entstehen. 


\section{Passive Lage und Bewegung:}

Die Wahrnehmung der passiven Lage und Bewegung nach Durchtrennung der periphereu Nerven zeigt die Integrität des tiefen Systems der Sensibilität. Dies benötigt dabei die Erhaltung anderer Formen von tiefer Sensibilität, nämlich die von Druck und von übermässigem Druck herrührenden Schmerz. Denn alle drei Formen von Sensibilität sind zusammen vorhanden oder fehlen nach Läsionen der peripheren Nerven. Wie oben gesagt, hängen alle drei von der Integrität jener afferenten Fasern ab, welche mit den Muskelnerven laufen.

Bei einer intramedullären Läsion kann jedoch jede von diesen drei Gruppen unabhängig von den beiden anderen affiziert werden (Schmerz, Druck, passive Lage und Bewegung) und wenn eine von diesen Empfindungen etwa abgeschwächt wird, dann werden alle Formen von Impulsen, welche das besondere System versorgen, geschwächt. Forner zeigen viele Fälle von intramedullärer Läsion, dass in einem bestimmten Areal oder Arealen Empfindung für passive Lage und Bewegung vollkommen fehlen kann und doch Druck oder Schmerzimpulse sofort und genau erkannt werden. Dies ist unmöglich bei einer (nur) von den peripheren Nerven stammenden Läsion.

\section{Die Zirkelprobe:}

Einer der bemerkenswertesten Unterschiede zwischen dem Verlust der Empfindung, der durch Durchtrennung eines peripheren Nervens erzeugt wird und dem, der von einer intramedullären Läsion des Rückenmarks herstammt, wird durch die Zirkelprobe offenbart. In bezug auf das periphere System zerstört eine Läsion, die einem peripheren Nerv Eintrag tut, sofort die Sensibilität für leichte Berührung und pari passu nimmt damit die Genauigkeit des Erkennens der beiden Spitzen des Zirkels beträchtlich bis unter normal ab. Wenn aber die Läsion innerhalb des Rückenmarks liegt, kann die Empfindlichkeit, leichte Berührung zu erkennen und das Vermögen, zwei Spitzen zu unterscheiden, dissoziiert sein. So zeigten Hoad und Thompson (13) in Fällen von intraspinaler Krankheit Areale, die empfindlich gegen leiseste und leichteste Berührung mit Baumwolle waren, die Zirkelspitzen aber nicht als zwei erkannten, wenn sie nicht bis zu einer Distanz von $15-20 \mathrm{~cm}$ getrennt waren, während in dem entsprechenden gesunden Areal der entgegengesetzten Seite der Patient richtige Antworten bei einer Entfernung von $1.5 \mathrm{~cm}$ gab. Daher sind im Rückenmark die Impulse, welche dem Vermögen, zwei Punkte zu unterscheiden, zugrunde liegen, von jenen der Tastunempfindlichkeit dissoziiert. Dies ist unmöglich bei einer Läsion nur der peripheren Nerven.

Aus obigen Tatsachen wird man ersehen, dass Schmerzimpulse das Rückenmark und das Sensorium durch das protopathische System und durch 
das System der tiefen Sensibilität (Druckschmerz) erreichen können, und dass eines von diesen Systemen unabhängig von dem anderen affiziert werden kann. Wenn aber diese Impulse das Rückenmark erreichen, werden alle Schmerzimpulse, von welcher Quelle sie auch herrühren mögen, von diesen beiden Systemen in ein einziges System oder eine einzige Bahn gelenkt, die nur dem Transport von schmerzhaften Impulsen zu dienen hat, so dass eine Läsion des Rückenmarks, welche das Erkennen der Haut applizierten schmerzhaften Reize schwächt oder vernichtet, notwendigerweise ebenfalls Druckschmerz auch vernichten muss, und vice versa.

Möglicherweise ist das Resultat der Gruppierung im Rückenmark von Wärme- und Kälteimpulsen noch auffallender. Es wurde von mehreren Forschern gezeigt, dass gelegentlich (wenn auch selten) Fälle entstehen, bei welchen eine Läsion des Rückenmarks die Leitung von Wärme und nicht die von Kälte und umgekehrt stören kann. Wenn ferner dank der Gruppierung im zentralen Nervensystem, im Fall dass dort eine Läsion ist, das Erkennen der Haut applizierten äussersten Hitze (oder Kälte) veruichtet ist, dann müssen auch die mittleren, warm genannten Temperaturen $24^{\circ}-40^{\circ}$ vernichtet werden und umgekehrt. Ein Hinweis auf das nebenstehende Diagramm mag zur Erläuterung dieser Tatsachen dienen.

Eine zusammenfassende Darstellung der Impulse, welche von diesen drei Arten von Sensibilitäten in den peripheren Nerven transportiert werden, und ihre Gruppierung beim Eintritt in die Medulla des Rückenmarks möge (mit Erlaubnis von $\mathrm{H}$ ead und seinen Mitarbeitern) folgendermassen teilweise und schematisch skizziert werden (siehe gegenüberstehend).

\section{Schlïisse.}

Eine grosse Anzahl von wichtigen und neuen Tatsachen, die von den verschiedenen Forschern zutage gefördert wurden, sind im obigen Aufsatze betrachtet worden. Aber unter diesen sind eine gewisse Anzahl, nicht die am wenigsten bedeutenden, von einer Gruppe von Forschern, die jahrelang auf koordinierte Weise arbeiteten, erhalten worden. Aus dem Studium der klinischen Fälle und aus dem Studium der Ergebnisse der Durchtrennung eines rein afferenten Hautnerven in Heads Arm, ist es Head, Rivers und Sherren intra alia gelungen, im afferenten System das Vorhandensein von drei peripheren Nervenmechanismen zu beweisen, nämlich die tiefe, protopathische und epikritische Sensibilität.

1. Die tiefe Sensibilität versorgt die Reaktion auf Druck, Druckschmerz, Örtlichkeit und Richtung der aktiven und passiven Bewegung; selbst wenn die Haut des Teiles durch Durchtrennung aller Hautnerven, welche das Integument versorgen, vollständig gefübllos gemacht worden ist. 

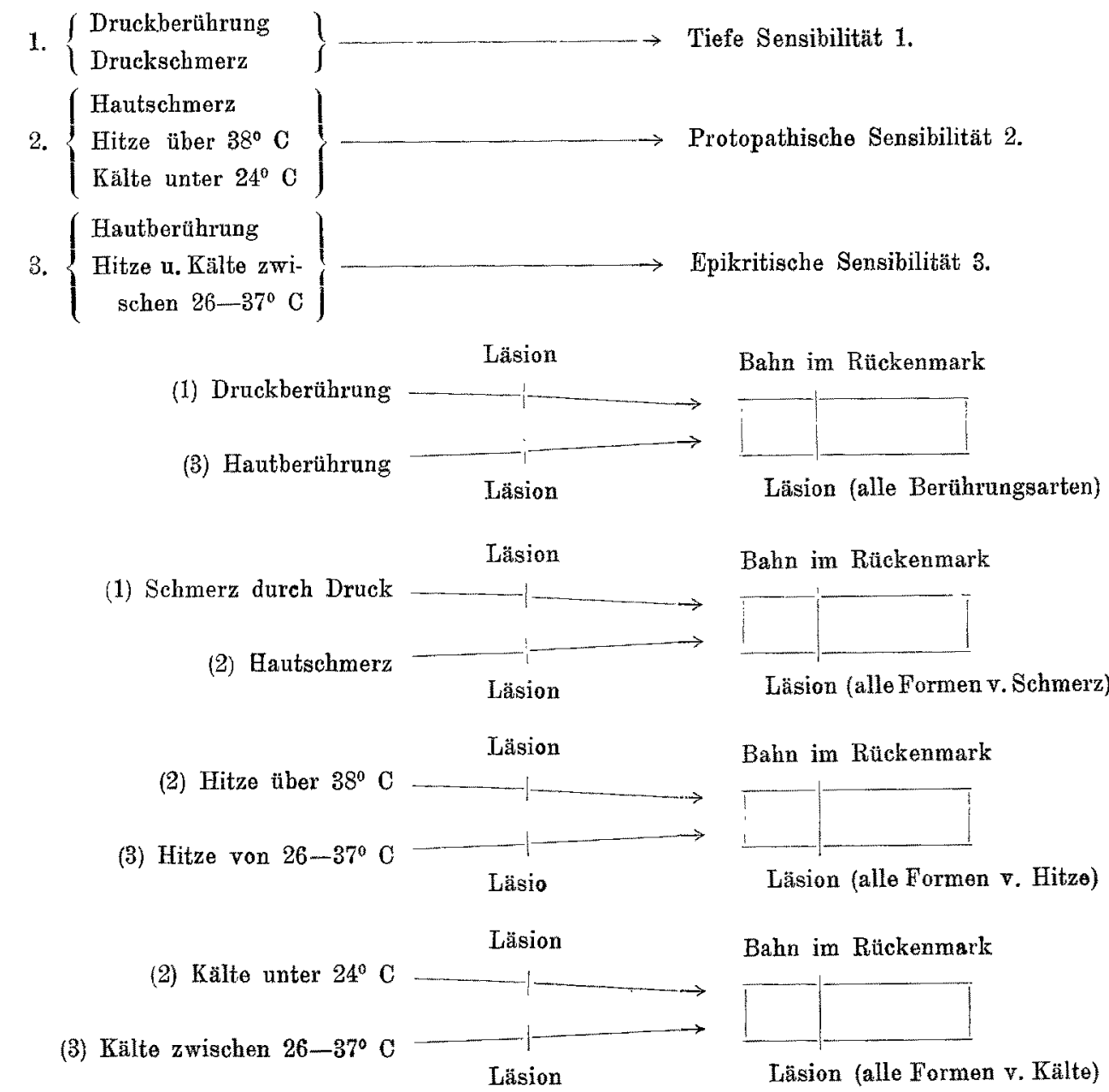

2. Die protopathische Sensibilität, welche zwischen 7 und 26 Wochen regeneriert, ermöglicht die Reaktion auf Schmerz, Hitze über ungefähr $38^{\circ} \mathrm{C}$, Kälte unter ungefähr $24^{\circ} \mathrm{C}$ und das Erkennen von Räue und einer diffusen, ungenauen Lokalisation. Jede erzeugte Empfindung besitzt einen besonderen stechenden und irradiierenden Charakter, der beim Auftreten der epikritischen Sensibilität verschwindet. Ferner ist die protopathische Sensibilität wesentlich auf eine punktförmige Anordnung basiert und veranlasst lebhafte Empfindungen.

3. Die epikritische Sensibilität regeneriert zwischen 1 bis 2 Jahren nach Durchtrennung des Hautnerven und entfernt sofort das diffusse Stechen und die lebhaften Eigenschaften des vorangehenden. Sie ermöglicht ein Erkennen jener Impulse, welche (leichter) Hautberührung, der gleich- 
zeitigen Applikation von zwei Punkten, feinen Temperaturgraden, die gewöhnlich warm oder kühl genannt werden ( $\mathrm{d}$. h. zwischen $26^{\circ}-37^{\circ} \mathrm{C}$ ), Grösse und einer feinen und genauen Lokalisation unterliegen.

4. Head und Thompson haben gezeigt, dass die verschiedenen Formen der spezifischen Impulse, welche in die tiefe, protopathische und epikritische Sensibilitäten eingehen, beim Eintritt in das zentrale Nervensystem regruppiert werden. So vereinigen sich Schmerzimpulse, welche in das tiefe System gehen, mit jenen des Schmerzes, welche in das protopathische System führen, so dass eine medulläre Läsion des Rückenmarks, die den Druckschmerz vernichtet auch den Schmerz, ob er von der Haut oder einem anderen Ursprung kommt, vernichtet; - alle Arten von Schmerzimpulsen jedweden Ursprunges aus einer Rückenmarksläsion werden gleich geschwächt oder vernichtet. Ähnlicherweise vereinigen sich die Impulse im protopathischen System, welche äusserster Hitze und Kälte unterliegen, im Rückenmark mit jenen, welche den mittleren Temperaturen $\left(26^{\circ}-37^{\circ}\right)$ unterliegen; denn wo Hitze überhaupt affiziert wird, ist es für alle Formen und Grade von Hitze, und wenn Kälte affiziert wird, ist es für alle Formen und Grade von Kälte.

Die oben erwähnten Forscher haben auch ein helles Licht auf viele früher dunkle oder falsch verstandene Punkte geworfen. Wenn z. B. es sich schon zeigt, „dass Zerstörung aller sensorischen Nerven nach der Haut, den Teil auf die meisten, gewöhnlich von dem Arzt und Chirurgen als eine Probe für Sensibilität für Berührung benutzten Reize, reaktionsfähig lässt", und wenn ,sie sagen, dass ein Stich mit einer Nadel gefühlt und gut lokalisiert würde, keinerlei Beweis für das Vorhandensein der Hautsensibilität gegen Schmerz ist", dann ist eine Erklärung erforderlich.

\section{Anmerkung:}

Denn diese beiden Behauptungen scheinen vielen Klinikern und anderen revolutionär zu sein, oder sie würden es vielmehr vor kurzem noch jedem so erschienen sein. Die Erklärung (wie sie oben gegeben ist) dieser beiden Tatsachen allein, ebenso wie viele andere im Text aufgestellte Behauptungen, klären viele der zahllosen von mehreren früheren Forschern erhaltenen sich widersprechende Resultate auf. Es wäre leicht, auf der direkten Anwendung dieser Tatsachen Massen von Resultaten, die in der medizinischen Literatur beschrieben worden sind, aufzuhäufen; aber Raum und Zeit sind beschränkt, und vielleicht würde es nützlicher sein, die zahlreichen, oben angegebenen Resultate in Verbindung mit der Erforschung der Empfindung, sei sie normal oder krank, in Zukunft zu betrachten.

Ohne in irgend welche spekulative Anschauung einzugehen, helfen obige Betrachtungen nicht nur bei der Diagnose und Prognose, sondern sie werfen ein helles und notwendiges Licht auf den ganzen Mechanismus der Empfindung. Bei klinischen Fällen kann man jedoch kaum die Tatsache zur selhr 
betonen, dass (S. 663) um Schwierigkeiten zu vermeiden, die Schwelle einen nicht zu geringen Umfang erhalten und einige der Bemerkungen über Methoden (S. 662) nicht übersehen werden sollten. Ferner ist es klar, dass Krankheit wie Verletzung nicht notwendigerweise alle afferenten Fasern durchtrennt, sie kann besonders dem frühen Stadium die Funktion einer variierenden Anzahl von Fasern, welche einen Nerven oder eine spinale $W^{\prime}$ urzel bilden, nur in einem variierenden Grade schwächen. Überdies kann mit der Zeit eine gewisse Menge von Regeneration stattgefunden haben, so dass in einem klinischem Fall in einem gegebenen Moment ein gemischtes und höchst komplexes Problem zur Lösung dargeboten wird. Die Gegenwart von Hyperästhesie und Hypoästhesie verschiedener Arten und Grade kann in demselben Teile mehr oder weniger vermischt gefunden werden. 